Article

\title{
A Two-Stage Multistep-Ahead Electricity Load Forecasting Scheme Based on LightGBM and Attention-BiLSTM
}

\author{
Jinwoong Park (1) and Eenjun Hwang *(i) \\ School of Electrical Engineering, Korea University, 145 Anam-ro, Seongbuk-gu, Seoul 02841, Korea; \\ timeless@korea.ac.kr \\ * Correspondence: ehwang04@korea.ac.kr; Tel.: +82-2-3290-3256
}

Citation: Park, J.; Hwang, E. A Two-Stage Multistep-Ahead

Electricity Load Forecasting Scheme Based on LightGBM and Attention-BiLSTM. Sensors 2021, 21, 7697. https://doi.org/10.3390/ s21227697

Academic Editors: Bruno Marhic and Laurent Delahoche

Received: 6 October 2021

Accepted: 17 November 2021

Published: 19 November 2021

Publisher's Note: MDPI stays neutral with regard to jurisdictional claims in published maps and institutional affiliations.

Copyright: (c) 2021 by the authors. Licensee MDPI, Basel, Switzerland. This article is an open access article distributed under the terms and conditions of the Creative Commons Attribution (CC BY) license (https:// creativecommons.org/licenses/by/ $4.0 /)$.

\begin{abstract}
An efficient energy operation strategy for the smart grid requires accurate day-ahead electricity load forecasts with high time resolutions, such as 15 or $30 \mathrm{~min}$. Most high-time resolution electricity load prediction techniques deal with a single output prediction, so their ability to cope with sudden load changes is limited. Multistep-ahead forecasting addresses this problem, but conventional multistep-ahead prediction models suffer from deterioration in prediction performance as the prediction range is expanded. In this paper, we propose a novel two-stage multistep-ahead forecasting model that combines a single-output forecasting model and a multistep-ahead forecasting model to solve the aforementioned problem. In the first stage, we perform a single-output prediction based on recent electricity load data using a light gradient boosting machine with time-series cross-validation, and feed it to the second stage. In the second stage, we construct a multistep-ahead forecasting model that applies an attention mechanism to sequence-to-sequence bidirectional long short-term memory (S2S ATT-BiLSTM). Compared to the single S2S ATT-BiLSTM model, our proposed model achieved improvements of $3.23 \%$ and $4.92 \%$ in mean absolute percentage error and normalized root mean square error, respectively.
\end{abstract}

Keywords: smart grid; electricity load forecasting; multistep-ahead forecasting; light gradient boosting machine; attention mechanism

\section{Introduction}

A smart grid is an efficient and intelligent energy operation solution that integrates traditional power systems and information and communications technology to enable two-way communication between consumers and suppliers [1]. Typical smart grids utilize renewable energy resources such as solar photovoltaic and wind power, and operate an energy management system to establish efficient energy operation plans through short-term energy load and supply forecasting. In particular, to establish elaborate energy operation strategies, a high time resolution, such as 15 or $30 \mathrm{~min}$, is essential in short-term load forecasting (STLF) [2]. Furthermore, STLF can be used to establish peak load response or energy storage system operation strategies to reduce energy costs. STLF is becoming increasingly important as it contributes to the implementation of stable power systems through the proper balance between supply and demand $[3,4]$.

So far, many STLF models have been proposed based on various methods [5]. For instance, statistical models for STLF include autoregressive [6], regression analysis [7], and holt winters [8]. These models perform well when the input and output are linear, but degrade when the input and output are nonlinear [9,10]. To address this concern, artificial intelligence (AI)-based models such as support vector regression (SVR) [11], neural networks (NN) [12], and extreme learning machines (ELMs) [13] have been proposed for single-point prediction. Even though such single-output forecasting models perform well even for nonlinear data, they show limited ability to cope with sudden electricity load changes [14]. To compensate for this, various multistep-ahead (MSA) forecasting 
models have been proposed using recurrent neural networks (RNNs), such as long shortterm memory (LSTM) [15] and gated recurrent unit (GRU) [16], that reflect time series characteristics satisfactorily. The performance was further improved by using a sequence-tosequence (S2S) architecture combining an encoder RNN and a decoder RNN [17]. However, information loss problems arose in S2S architecture RNN models because the encoder compresses all necessary information into a fixed-length vector. To tackle this problem, attempts have been made to apply an attention mechanism to forecasting models so that the decoder can look back on the most relevant information from the encoder output $[18,19]$. However, this approach showed performance deterioration as the forecasting horizon was expanded [20].

In this paper, we propose a two-stage MSA electricity load forecasting model to deal with the aforementioned issue. The forecasting horizon is one day from the current time at 15 min intervals, which gives 96 time points. In the first stage, a single-output forecasting model performs day-ahead prediction. The forecasting model is constructed using light gradient boosting machine (LightGBM) and time-series cross-validation (TSCV). In the second stage, an MSA forecasting model based on an S2S bidirectional LSTM with attention mechanism (ATT-BiLSTM) performs predictions for 96 time points using the output of the first-stage forecasting model and other external data. The contributions of this paper are as follows:

- We present a forecasting model that combines an ensemble learning method and an RNN for accurate MSA forecasting;

- We show that the performance of an MSA forecasting model can be further improved by considering the prediction result of a single-output forecasting model;

- The proposed model shows very stable forecasting accuracy over the entire forecasting horizon of 96 time points at 15 min intervals.

This paper is organized as follows. Section 2 introduces some related works. Section 3 describes data collection and preprocessing. Section 4 presents the overall structure of the proposed two-stage MSA forecasting model. Section 5 illustrates the experiments and their results. Finally, Section 6 concludes the paper.

\section{Related Works}

Recently, forecasting models using AI methods such as artificial neural networks (ANNs) [21,22] and SVR [23] have been proposed to overcome nonlinearities and complex relationships of time series. For instance, Grolinger et al. [24] constructed a forecasting model based on ANN and SVR to accurately forecast the electricity load of an entertainment building. Jurado et al. [25] predicted the hourly electrical loads of three teaching buildings using various machine learning (ML) methods such as random forest (RF), ANN, fuzzy inductive reasoning (FIR), and autoregressive integrated moving average (ARIMA). Zhang et al. [26] proposed an SVR-based electricity load forecasting model and optimized the hyperparameters using the Cuckoo search algorithm.

On the other hand, Zheng et al. [27] proposed an LSTM-based short-term electricity load forecasting model and showed that an LSTM-based forecasting model can predict complex univariate electricity loads with non-stationarity and non-seasonality. Marino et al. [28] also constructed two electricity load forecasting models based on the standard LSTM and the S2S architecture LSTM, and compared their performance. Both models were trained and tested for one-hour and one-minute time resolution data. They showed that the standard LSTM was unable to accurately forecast loads for one-minute resolution, but the S2S architecture LSTM performed well for one-hour and one-minute time resolution datasets.

Even though these single-output forecasting models have demonstrated good prediction accuracy, they are limited in handling uncertainties, such as sudden load changes. To address this issue, various MSA models have been proposed by simultaneously predicting multiple time points. For instance, Kim et al. [29] proposed a recurrent inception convolution neural network (RICNN) for MSA electricity load forecasting (48 time points at $30 \mathrm{~min}$ 
intervals). They combined the RNN and the 1-D convolution inception to calibrate the forecasting time and hidden state vector values calculated from the nearby forecasting time points. Jung et al. [30] proposed an MSA load forecasting model (24 time points at $1 \mathrm{~h}$ intervals) using a GRU network with attention mechanism. They demonstrated that an attention mechanism can improve the forecasting performance of RNNs by $10 \%$ or more. Kuo et al. [31] proposed a one-dimensional CNN-based power load prediction model with three convolution layers. Despite their advantages, these works show limited performance improvement because they use a single prediction algorithm.

Various studies have been conducted to overcome this limitation by combining multiple forecasting models. For instance, Park et al. [32] proposed a two-stage STLF model. The first stage consists of extreme gradient boosting (XGB) and RF methods for prediction, and the second stage combines their predictions using a sliding window-based multiple linear regression (MLR) model. Siridhipakul et al. [33] proposed a dual-stage attentional LSTM (DALSTM), which is a two-stage MSA electricity load-forecasting (48 time points at 30 min intervals) model based on LSTM and a dual-stage attention-based recurrent neural network (DARNN). The first stage performs MSA prediction using LSTM, and the second stage performs another MSA prediction based on the DARNN using the prediction values from the first stage and the other inputs. Moon et al. [34] proposed an MSA forecasting model using sliding window-based principal component regression (PCR), performing single-output forecasting in the first stage using four MLP models with different layers and then inputting their prediction values in the second stage. Nie et al. [35] proposed a hybrid forecasting model based on ARIMA and SVM. The model first predicted the electricity load preliminary for $24 \mathrm{~h}$ based on ARIMA, and then used SVM to correct the deviation from the previous prediction. Tian et al. [36] proposed a hybrid forecasting model based on LSTM and CNN. The model extracted the temporal features and local trend of the electricity load data, and used them to make final predictions. Xie et al. [37] proposed a two-stage forecasting model combining ATT-BiLSTM and MLP. First, $24 \mathrm{~h}$ prediction was performed based on ATT-BiLSTM, and then MLP-based prediction was performed using the result as an input.

Even though many LSTM-based methods showed strength in time series prediction, LSTM-based MSA electricity load forecasting methods have not solved the problem of performance degradation due to the increase in the prediction horizon [20]. Hence, to address this problem, we present a robust two-stage MSA forecasting model using a LightGBM-based day-ahead forecasting model and an S2S ATT-BiLSTM-based MSA forecasting model.

\section{Data Collection and Preprocessing}

In this section, we describe the data collection and preprocessing that we performed to configure and train our forecasting model. We collected the electricity load data of buildings of a private university in Seoul, Korea at 15 min intervals from the Korea Electric Power Corporation through the i-Smart system. We classified the university buildings into four clusters based on their use and location. Cluster A consists of humanities and social sciences buildings, while clusters B and D are both science/engineering buildings and their laboratories. Finally, cluster $C$ is dormitories and sports facilities. The data were collected for 67 months from 1 January 2015 to 31 July 2020 for clusters A, B, and C, and 58 months from 1 September 2015 to 31 July 2020 for cluster D. In addition, we considered calendar information, weather data, and historical load data as input variables to the load forecasting model. The details are described in the following subsections.

\subsection{Weather Data}

We used the weather data provided by the Korea Meteorological Administration (KMA). The KMA provides weather forecasts for most major areas, including short-term forecasts such as the Dong-Nae Forecast and mid-term forecast. Short-term forecasts provide weather forecasts every three hours for three days from the present day. As our 
goal is to construct a day-ahead load forecasting model, we collected weather data for the same area as the clusters using the short-term weather forecast. The short-term forecast provides sky conditions, temperature, humidity, wind speed, wind direction, precipitation, and other information. As the predictions are quite accurate, there is little difference between the forecast data and the measured data. An example of the Dong-Nae forecast is illustrated in Figure 1.

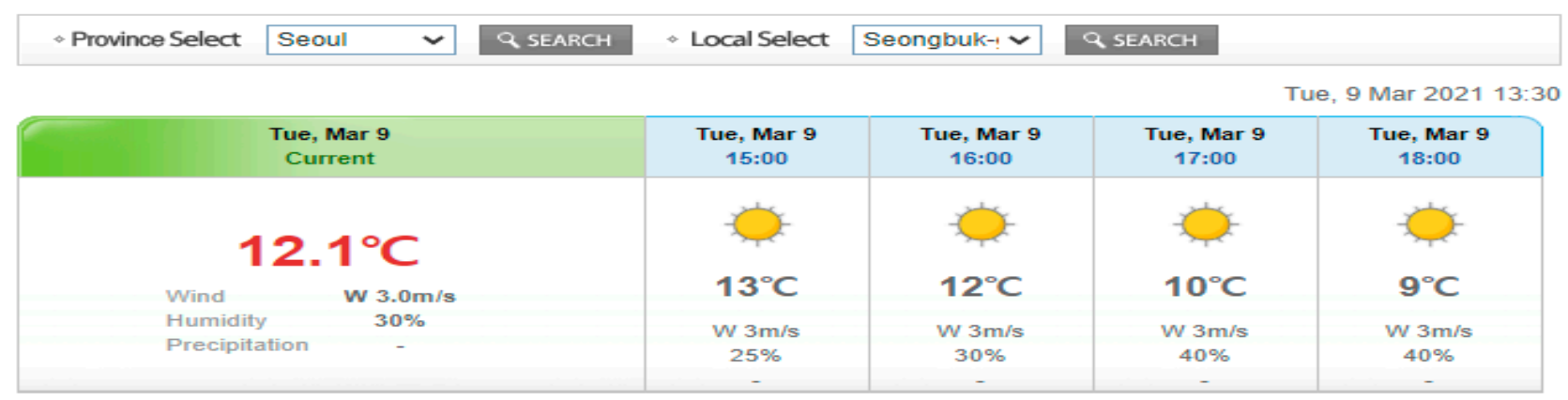

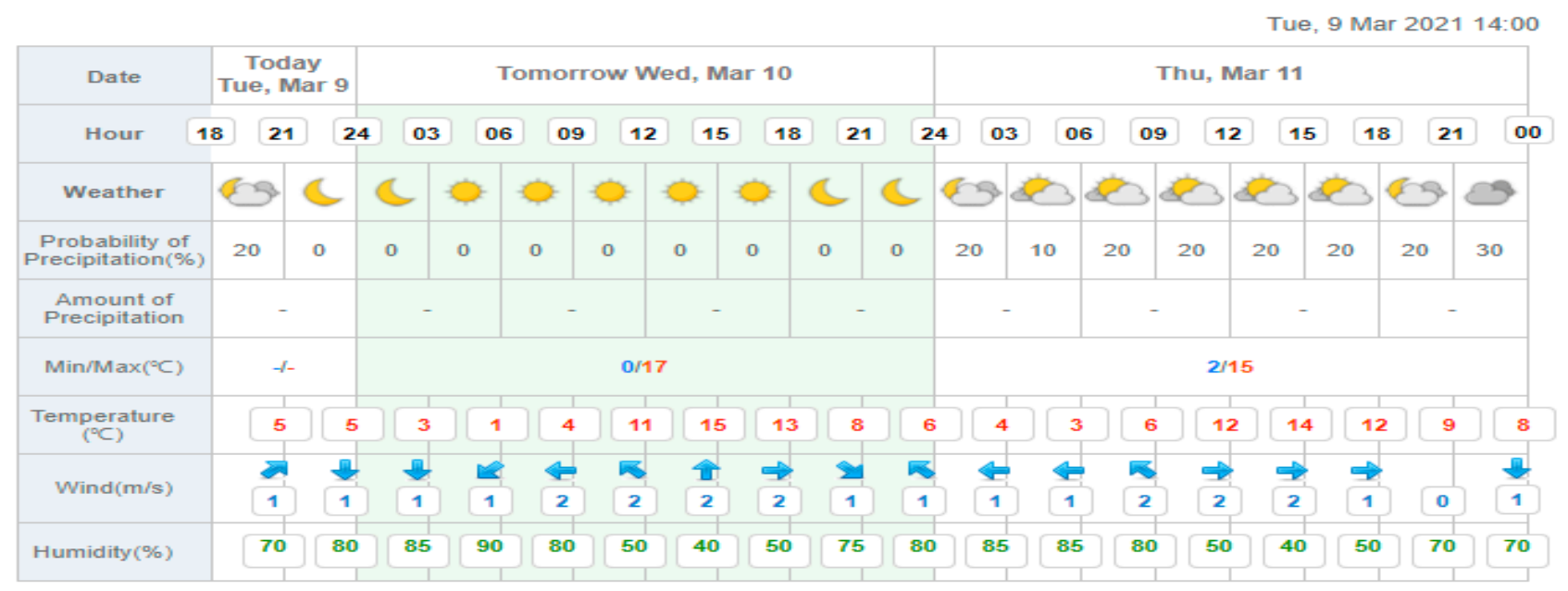

Figure 1. Example of Dong-Nae forecast by the Korea Meteorological Administration.

In this study, we considered four types of weather data: temperature, humidity, wind speed, and wind direction. Those weather data were provided by KMA's short-term weather forecast as illustrated in Figure 1 [29]. Among them, weather data were provided at $3 \mathrm{~h}$ intervals. To construct a forecasting model at $15 \mathrm{~min}$ intervals, we estimated the weather data at a $15 \mathrm{~min}$ resolution using linear interpolation in Equation (1) [34].

$$
f(T)=\frac{d_{2}}{d_{1}+d_{2}} f\left(T_{1}\right)+\frac{d_{1}}{d_{1}+d_{2}} f\left(T_{2}\right)
$$

The equation calculates the data value $f(T)$ at any point $T$ between two points $T_{1}$ and $T_{2}$ where $T_{1}$ and $T_{2}$ have data values $f\left(T_{1}\right)$ and $f\left(T_{2}\right)$, respectively. Here, $d_{1}$ is the distance between $T$ and $T_{1}$ and $d_{2}$ is the distance between $T$ and $T_{2}$. In addition, two indices were calculated to reflect the weather the human body actually feels in the model: windchill index (WCI) for the actual temperature and discomfort index (DI) for feeling hot or cold [38]. WCI and DI are calculated using Equations (2) and (3), respectively.

$$
\begin{gathered}
W C I=(10 \sqrt{v}-v+10.5) \times\left(33-T_{a}\right) \\
D I=T-0.55 \times(1-0.01 H) \times(T-14.5)
\end{gathered}
$$


Here, $v$ and $T_{a}$ indicate wind speed in $\mathrm{m} / \mathrm{s}$ and temperature in degrees Celsius, respectively, and $T$ and $H$ represent temperature in degrees Celsius and relative humidity in $\%$, respectively.

\subsection{Calendar Information and Historical Electricity Load}

As the electricity load data are time-series data, calendar information is essential in electricity load forecasting. For calendar information, we considered months, days, hours, minutes, day of the week, and holidays, and used them as input variables of the forecasting model. Time data, including month, day, hour, and minute, are represented as numeric, and day of the week data is defined as 0 to 6 from Monday to Sunday. Holiday data are represented by one-hot encoding (i.e., " 1 " for holiday, otherwise " 0 "). We consider historical electricity load data as the input variables so that the forecasting model can reflect the recent trends in electricity load [34]. Historical electricity load data play an important role in accurate forecasting as they reveal recent electricity load patterns and trends [2]. Therefore, we selected historical electricity load data from seven days to one day before the same point as the forecast time as input variables. Table 1 presents the 19 input variables that we used to construct the forecasting model, and Figure 2 illustrates the Pearson correlation coefficients (PCCs) between the input variables and the electricity load, excluding calendar information.

In this figure, the past load of 1 day and 7 days ago shows a strong correlation with the actual electricity load. The remaining input variables are also positively related to the electrical load.

Table 1. List of input variables for the proposed model.

\begin{tabular}{|c|c|c|c|}
\hline $\begin{array}{l}\text { Input Variable } \\
\text { Identifier }\end{array}$ & Description (Type) & $\begin{array}{l}\text { Input Variable } \\
\text { Identifier }\end{array}$ & Description (Type) \\
\hline No.01 & Month (numeric) & No.11 & Windchill index (numeric) \\
\hline No.02 & Day (numeric) & No.12 & Discomfort index (numeric) \\
\hline No.03 & Hour (numeric) & No.13 & $\mathrm{D}-7$ same point load (numeric) \\
\hline No.04 & Min (numeric) & No.14 & $\mathrm{D}-6$ same point load (numeric) \\
\hline No.05 & Day of the week (numeric) & No.15 & $\mathrm{D}-5$ same point load (numeric) \\
\hline No.06 & Holiday (binary) & No.16 & $\mathrm{D}-4$ same point load (numeric) \\
\hline No.07 & Temperature (numeric) & No.17 & $\mathrm{D}-3$ same point load (numeric) \\
\hline No.08 & Humidity (numeric) & No.18 & D - 2 same point load (numeric) \\
\hline No.09 & Wind speed (numeric) & No.19 & $\mathrm{D}-1$ same point load (numeric) \\
\hline No.10 & Wind direction (numeric) & & \\
\hline
\end{tabular}




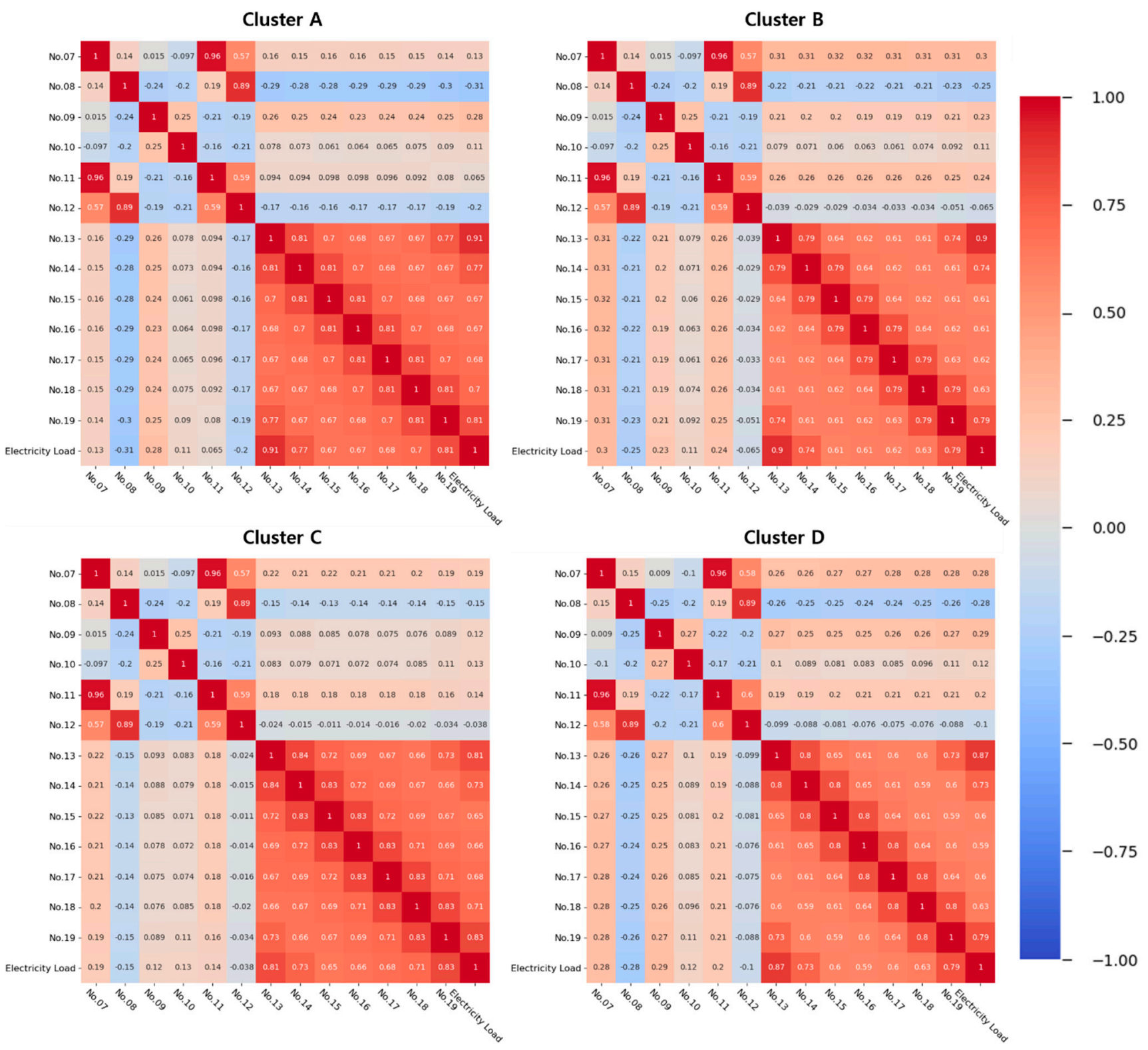

Figure 2. PCCs between input variables except calendar data and electricity load.

\section{Methodology}

In this section, we describe our two-stage MSA electricity load forecasting model. Figure 3 illustrates the overall architecture of the model. In the first stage, the LightGBMbased forecasting model performs a single-output prediction. LightGBM is a popular ensemble learning algorithm. In the second stage, a forecasting model based on bidirectional LSTM of the S2S architecture and the attention mechanism performs MSA prediction. We used data from January 2015 to December 2018 as the training set and data from January 2019 to July 2020 as the test set. The training set and test set are about $71.75 \%$ and $28.25 \%$ of the total dataset. The details are described in the following subsections. 


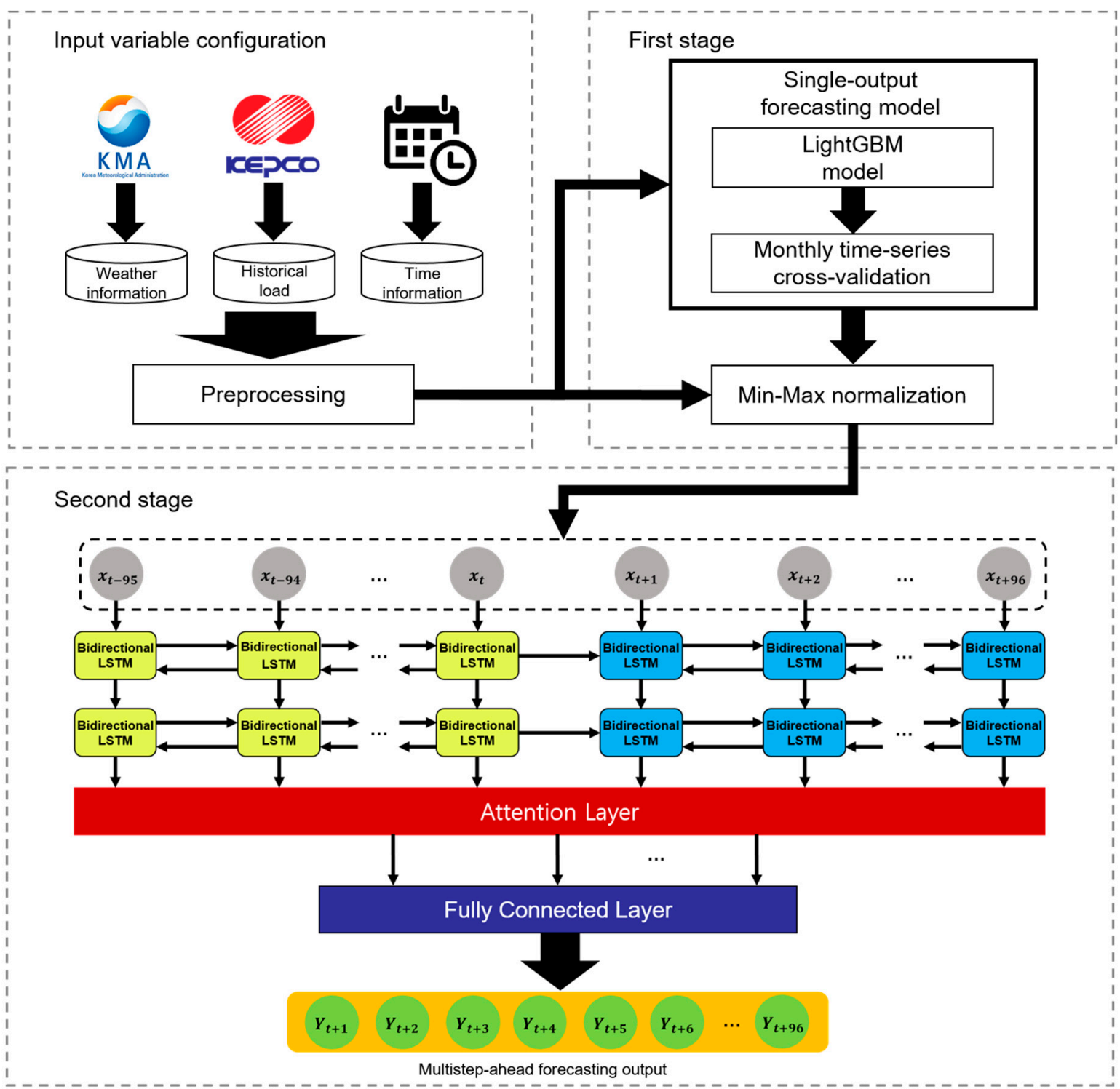

Figure 3. Architecture of two-stage multistep-ahead forecasting model.

\subsection{Single-Output Forecasting}

In the first stage, single-output forecasting is performed using a LightGBM-based forecasting model and the forecasting values are fed into the second stage as input variables. The model was trained using four years of data. Specifically, for training, data from the first year were used as training data and TSCV was performed on the data from the next three years. Sections 4.1.1 and 4.1.2 describe how we constructed single-output forecasting models.

\subsubsection{LightGBM}

LightGBM is a boosting-based algorithm that allows faster and more accurate forecasting compared to other boosting and bagging algorithms [39,40]. It is based on a gradient boosting decision tree (GBDT) with gradient-based one-sided sampling and exclusive feature-bundling technologies. Unlike the traditional gradient boosting machine (GBM) tree splitting method, LightGBM uses a leafwise method to achieve higher accuracy through more complex modeling. Therefore, it is better for time series forecasting, and owing to the GBDT and leaf method, LightGBM has the advantage of low memory usage and a fast training speed. LightGBM contains many hyperparameters, of which learning 
rate, number of iterations, and number of leaves are closely related to forecasting accuracy. In addition, LightGBM can prevent overfitting by adjusting colsample by tree and subsample hyperparameters. LightGBM has been used for various time series forecasting tasks, such as electricity load forecasting [41,42] and wind power forecasting [43], and its single-output forecasting has been proven to be fast and accurate. As we need a fast and accurate single-output forecasting model in the first stage, we construct it using LightGBM.

\subsubsection{Time Series Cross-Validation}

In general, to make a forecasting model, data are collected and split into a training set and a test set. The training set is used to construct the forecasting model, while the test set is used to evaluate its performance. Because single-output prediction is used as one of the inputs to the second stage forecasting model, our single-output forecasting model uses one year of training data. However, when the amount of training data is small, the accuracy decreases as the forecasting points get farther away [44]. To mitigate this concern, we used TSCV, which is a popular method when focusing on single-output forecasting in the data set when the data have time series characteristics [45]. TSCV uses all data before the forecasting point as a training set and forecasts the next forecasting point by setting it as a test set, iteratively. However, if TSCV is performed at every point in time, an enormous amount of time may be required for training and forecasting. To reduce this overhead, we used monthly TSCV, as illustrated in Figure 4.

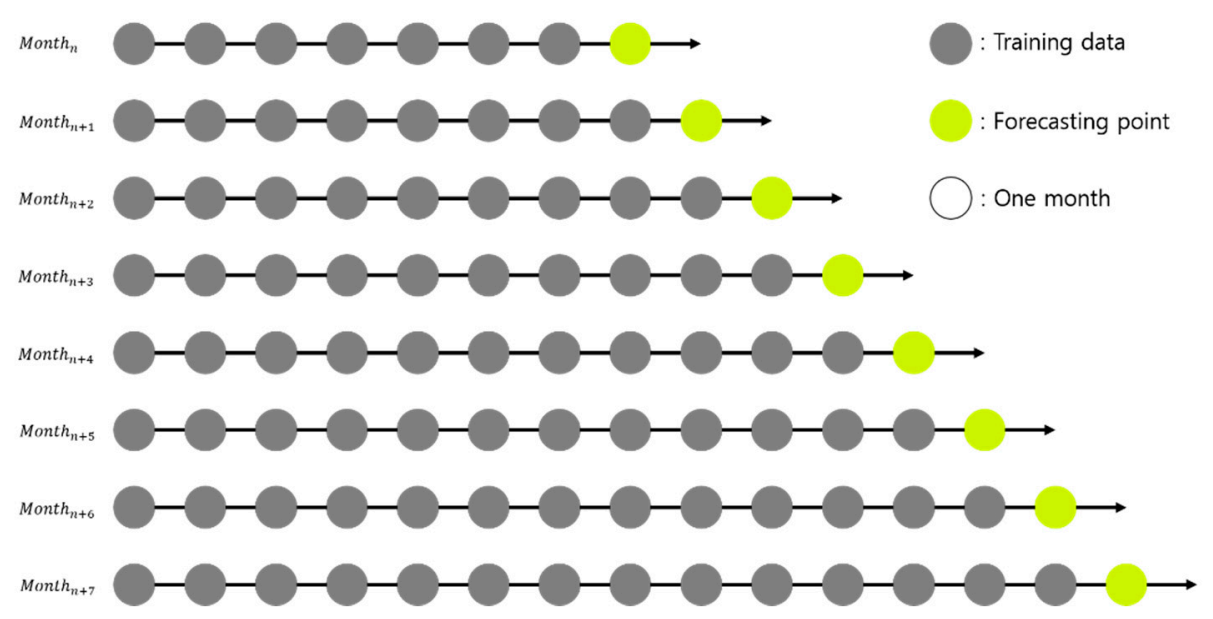

Figure 4. Example of monthly time-series cross-validation.

\subsection{Attention-BiLSTM Based MSA Forecasting}

In the second stage, we constructed an MSA forecasting model of the S2S architecture based on the attention mechanism and bidirectional LSTM. In addition to the configured input variables, the model used the values of the single-output forecasting model in the first stage as its input variable. In S2S bidirectional LSTM networks, information loss could occur because the encoder compresses all the information into a fixed-length vector. To avoid this, we applied an attention mechanism to the model.

\subsubsection{Bidirectional Long Short-Term Memory}

Traditional RNNs are trained using backpropagation through time [46]. However, RNNs can exhibit the vanishing gradient problem for longer sequences of inputs [47]. LSTM [48], which was designed to mitigate this issue, is made by adding three gates to each cell of traditional RNNs. The three gates are input, forget, and output. Figure 5 illustrates the architecture of the LSTM, and the LSTM cell calculation at time $t$ for input 
$x$ is given by Equations (4)-(9). Here, $g$ is an update step, and $c$ and $h$ indicate the cell memory state and hidden state, respectively.

$$
\begin{gathered}
f_{t}=\sigma\left(W_{h f} * h_{t-1}+W_{x f} * x_{t}\right) \\
i_{t}=\sigma\left(W_{h i} * h_{t-1}+W_{\varphi} * x_{t}\right) \\
g_{t}=\tanh \left(W_{h g} * h_{t-1}+W_{x g} * x_{t}\right) \\
o_{t}=\sigma\left(W_{h o} * h_{t-1}+W_{x o} * x_{t}\right) \\
c_{t}=f_{t} \odot c_{t-1}+i_{t} \odot g_{t} \\
h_{t}=o_{t} \odot \tanh \left(c_{t}\right)
\end{gathered}
$$

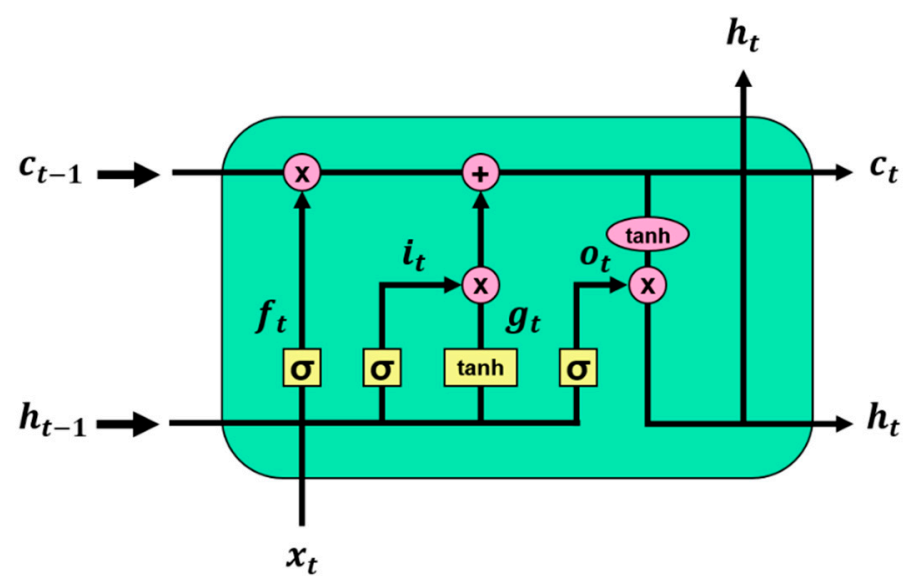

Figure 5. Architecture of long short-term memory.

LSTM stores the information of the input data in a hidden layer by adding the concept of time series. However, as the input information is stacked in the time sequence at the hidden layer, the most recent input information is reflected in the result [49]. Bidirectional LSTM (BiLSTM) is an extended version of the traditional LSTM that considers past and future states to improve forecasting performance [50]. BiLSTM processes data into two networks, i.e., the forward LSTM and the backward LSTM, and the outputs of the two networks are merged at each time step. The architecture of the BiLSTM is illustrated in Figure 6.

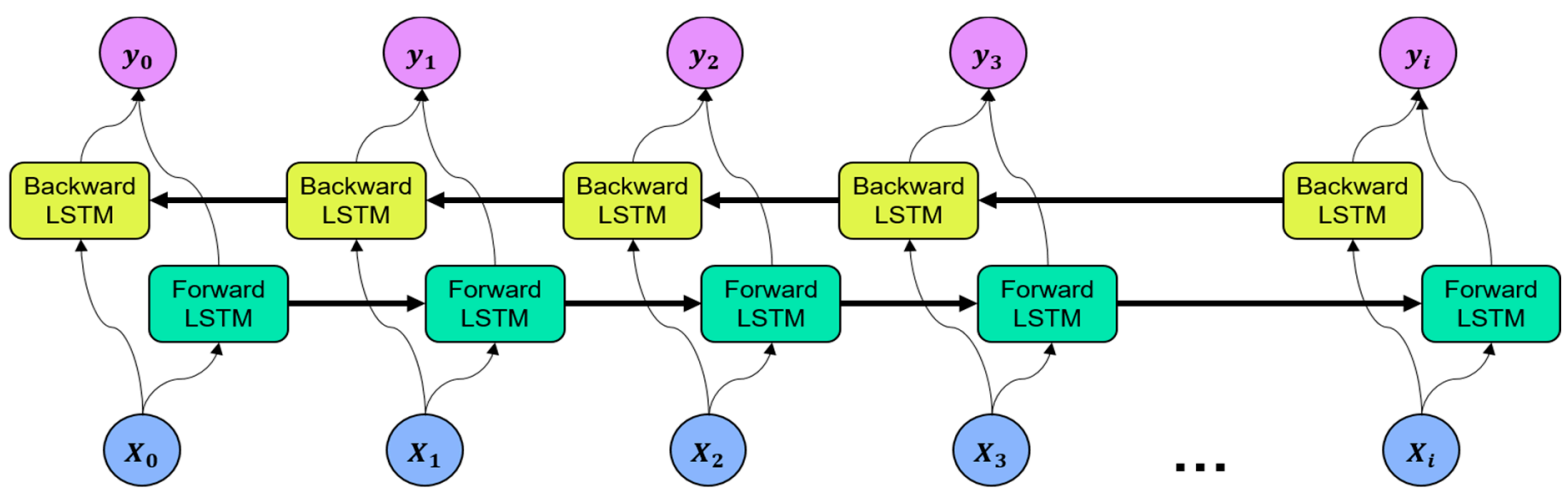

Figure 6. Architecture of bidirectional long short-term memory. 


\subsubsection{Sequence-to-Sequence Recurrent Neural Networks}

An S2S RNN contains two RNNs, an encoder and a decoder, as shown in Figure 7. The general idea is to pass an input sequence vector $x_{1}, x_{2}, \ldots, x_{T}$ one time step at a time to the encoder RNN to obtain a context vector. A common approach is to use an encoder RNN, as given by Equations (10) and (11).

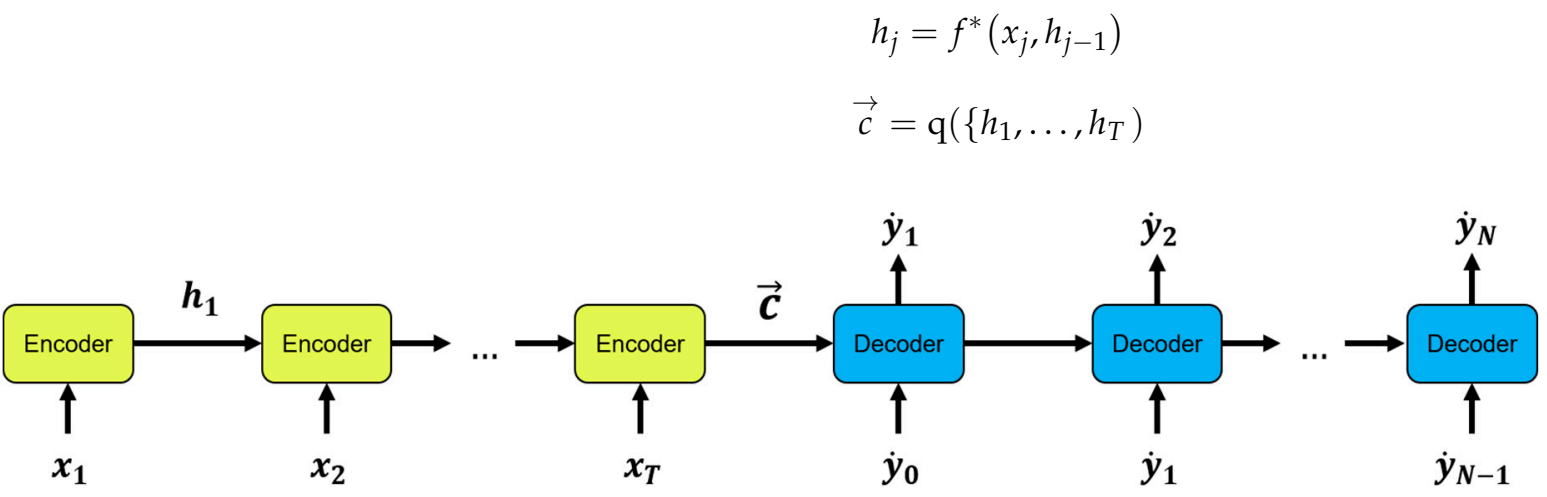

Figure 7. Sequence-to-Sequence recurrent neural network.

Here, $h_{j}$ is the hidden state at time $j$, and $f^{*}$ and q are nonlinear functions [51]. $\vec{c}$ is generated from a sequence of hidden states. The context vector is an encoded representation of the input sequence, passed to the decoder RNN, which extracts information at each unraveled time step to obtain the output sequence $\dot{y}_{1}, \ldots, \dot{y}_{N}$. The S2S output is obtained using Equation (12).

$$
\dot{y}_{N}=g^{*}\left(\dot{y}_{i-1}, \ldots, h_{i-1}^{*}\right)
$$

Here, $h_{i}^{*}$ is the hidden state of the decoder at time $i$ and $g^{*}$ is a nonlinear function. $\dot{y}_{0}$ is the context value (derived value from $\vec{c}$ ) used as the initial input to the decoder. The S2S RNN can enhance the continuous sequence forecasting and the temporal dimensions of the inputs and outputs. This can improve the performance of electricity load forecasting.

\subsubsection{Attention Mechanism}

In a model of S2S architecture, the encoder compresses all the information of the input sequence into a single context vector. For longer input sequences, the decoder has difficulties extracting valuable information from this single vector. To address this issue, attention mechanisms have been devised. Bahdanau et al. [18] and Luong et al. [19] each added attention mechanisms to the S2S model for machine translation. Machine translation is a very different task from predicting electricity loads. Nevertheless, various studies $[20,30]$ have shown that the attention mechanism benefits electricity load forecasting. Our model adopts the Bahdanau attention mechanism (BA) for electricity load forecasting. The S2S model with BA first obtains the hidden state of the current decoder at time $i$ using Equation (13). The forecasted value is then obtained using Equation (14).

$$
\begin{gathered}
h_{i}^{b}=f^{b}\left(\left[\dot{y}_{i-1} ; c_{i}^{b}\right]+h_{i-1}^{b}\right) \\
\dot{y}_{i}=g^{b}\left(\dot{y}_{i-1}, c_{i}^{b}, h_{i}^{b}\right)
\end{gathered}
$$

Here, $b$ denotes the variable used in $\mathrm{BA}$, and $i$ and $j$ denote the decoder and encoder variables, respectively. In the equation, $f^{b}$ takes the previous output $\dot{y}_{i-1}$, attention context vector $c_{i}^{b}$, and previous hidden state $h_{i-1}^{b} \cdot f^{b}$ and $g^{b}$ are the LSTM cell and nonlinear function, respectively. Each encoder output $h_{j}$ contains information for the $j$-th part of the 
input sequence. The vector $c_{i}^{b}$ is calculated as the weighted sum of the encoder outputs, as shown in Equation (15).

$$
c_{i}^{b}=\sum_{j=1}^{T} \alpha_{i j}^{b} h_{j}
$$

The attention weight $\alpha_{i j}^{b}$ of each encoder output $j$ is calculated using Equation (16).

$$
\alpha_{i j}^{b}=\frac{\exp \left(e_{i j}^{b}\right)}{\sum_{k=1}^{T} \exp \left(e_{i k}^{b}\right)}
$$

The attention scores $e_{i j}^{b}$ are calculated using Equation (17).

$$
e_{i j}^{b}=S\left(h_{i-1}^{b}, h_{j}\right)
$$

Here, $S$ is an alignment model that scores how well the inputs around time $j$ and the output at time $i$ match. Attention weight $\alpha_{i j}^{b}$ and attention score $e_{i j}^{b}$ reflect the importance of each encoder output $h_{j}$ when generating the next hidden state $h_{i}^{b}$ and forecasting value $\dot{y}_{i}$. This allows the decoder to pay attention to the important parts of the input sequence.

In the second stage, we combine the attention mechanism with the Sequence-toSequence BiLSTM and construct an S2S ATT-BiLSTM-based MSA forecasting model that performs MSA electricity load forecasting at 96 time points at $15 \mathrm{~min}$ intervals. To construct the BiLSTM network, we considered several hyperparameters. The input layer of the BiLSTM model consists of 20 nodes, and the hidden layer consists of 15 nodes per layer by applying $2 / 3$ of the input layer and the size of the output layer [34]. We used two layers for the number of hidden layers. The fully connected layer activation function uses a rectified linear unit (ReLU) to solve the gradient vanishing problem [52]. Huber loss [53] was used as the loss function, and adaptive moment estimation (Adam) [54] was used as the optimization algorithm. The learning rate and epochs were set to 0.001 and 350 .

\section{Results and Discussion}

As our forecasting model is composed of two stages, each with a different predictive model, we performed comparative experiments for each model. In the experiments, we used electricity load data collected from four building clusters of a private university in Seoul, Korea, at $15 \mathrm{~min}$ intervals through the i-Smart system. The clusters were determined based on their use and location. We first investigated the electric load characteristics of four clusters via box plot and various statistical analyses, as presented in Figure 8 and Table 2. For statistical analysis, we used the descriptive statistical data analysis tool of Excel.

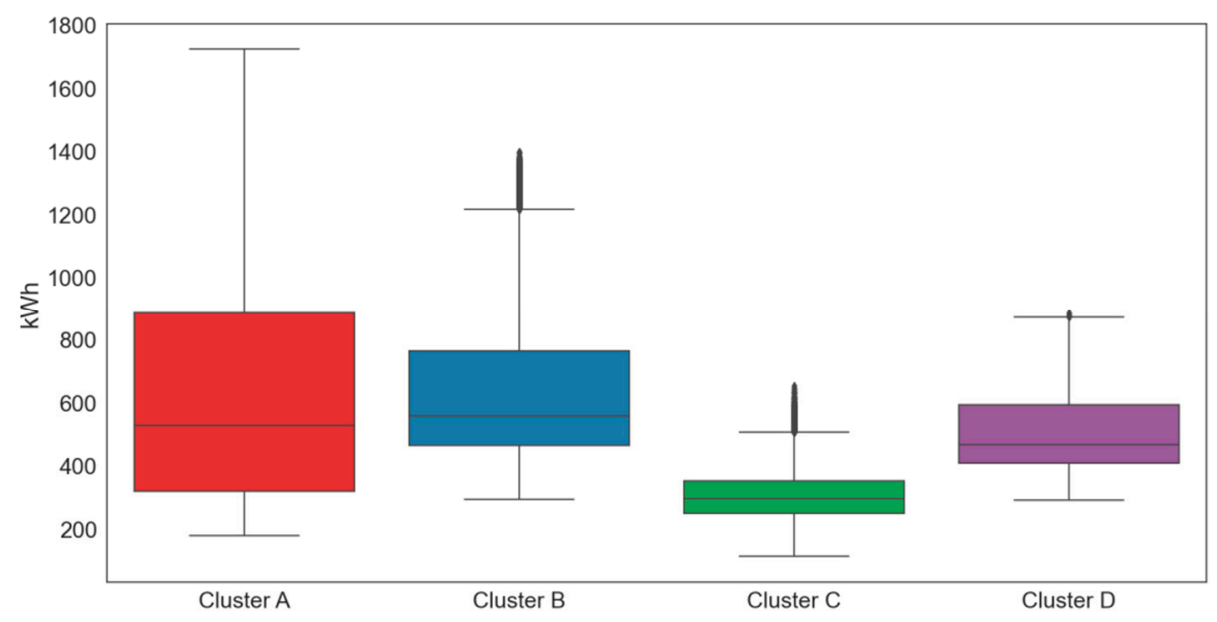

Figure 8. Boxplots by cluster (kWh). 
Table 2. Statistical analysis of electricity load data by cluster (kWh).

\begin{tabular}{|c|c|c|c|c|c|c|c|c|}
\hline & \multicolumn{2}{|c|}{ Cluster A } & \multicolumn{2}{|c|}{ Cluster B } & \multicolumn{2}{|c|}{ Cluster C } & \multicolumn{2}{|c|}{ Cluster D } \\
\hline & $\begin{array}{l}\text { Training } \\
\text { Set }\end{array}$ & $\begin{array}{l}\text { Test } \\
\text { Set }\end{array}$ & $\begin{array}{l}\text { Training } \\
\text { Set }\end{array}$ & $\begin{array}{l}\text { Test } \\
\text { Set }\end{array}$ & $\begin{array}{l}\text { Training } \\
\text { Set }\end{array}$ & $\begin{array}{l}\text { Test } \\
\text { Set }\end{array}$ & $\begin{array}{l}\text { Training } \\
\text { Set }\end{array}$ & $\begin{array}{l}\text { Test } \\
\text { Set }\end{array}$ \\
\hline Mean & 656.499 & 586.247 & 623.109 & 670.012 & 302.790 & 322.904 & 515.166 & 489.349 \\
\hline Standard error & 0.954 & 1.418 & 0.587 & 0.929 & 0.205 & 0.323 & 0.35 & 0.515 \\
\hline Median & 553.4 & 462.7 & 543.8 & 593.3 & 292.3 & 311.8 & 478.2 & 447.3 \\
\hline Mode & 271.2 & 265.9 & 454.1 & 518.4 & 259.9 & 275.8 & 413.4 & 414.9 \\
\hline Standard deviation & 357.686 & 333.674 & 220.187 & 218.575 & 76.902 & 76.172 & 119.92 & 121.328 \\
\hline Sample variance & $127,939.5$ & $111,338.3$ & $48,482.45$ & $47,775.23$ & 5914.042 & 5802.298 & $14,380.87$ & $14,720.47$ \\
\hline Kurtosis & -0.721 & -0.758 & 0.352 & 0.434 & 0.375 & 0.233 & -0.597 & -0.225 \\
\hline Skewness & 0.676 & 0.736 & 1.076 & 1.096 & 0.689 & 0.716 & 0.673 & 0.871 \\
\hline Range & 1529.7 & 1350.2 & 1104 & 1017.1 & 541.8 & 476.9 & 586.8 & 556.5 \\
\hline Minimum & 195.4 & 181.4 & 296.2 & 383.2 & 114.1 & 130.7 & 300.6 & 292.5 \\
\hline Maximum & 1725.1 & 1531.6 & 1400.2 & 1400.3 & 655.9 & 607.6 & 887.4 & 849 \\
\hline Sum & $92,204,063$ & $32,417,138$ & $87,514,440$ & $37,049,022$ & $42,526,339$ & $17,855,317$ & $60,336,352$ & $27,059,063$ \\
\hline Count & 140,448 & 55,296 & 140,448 & 55,296 & 140,448 & 55,296 & 117,120 & 55,296 \\
\hline
\end{tabular}

To reflect all data with the same degree of importance, the input data were preprocessed by min-max normalization, defined by Equation (18). In the equation, $x$ represents the original data, and $x_{\min }$ and $x_{\max }$ represent the minimum and maximum values of the original data, respectively. Finally, all the values are normalized to between 0 and 1 .

$$
x_{n o r m}=\frac{x-x_{\min }}{x_{\max }-x_{\min }}
$$

To evaluate the forecasting performance of the proposed model, we used four metrics: mean absolute error (MAE), mean absolute percentage error (MAPE), root mean square error (RMSE), and normalized root mean square error (NRMSE), as given in Equations (19)-(22). Here, $A_{t}$ and $F_{t}$ represent the actual and forecasted values, respectively, at time $t . n$ indicates the number of observations, and $\bar{A}$ represents the mean of the actual values.

$$
\begin{gathered}
M A E=\frac{1}{n} \sum_{t=1}^{n}\left|A_{t}-F_{t}\right| \\
M A P E=\frac{100}{n} \sum_{t=1}^{n}\left|\frac{\left|A_{t}-F_{t}\right|}{A_{t}}\right| \\
\text { RMSE }=\sqrt{\frac{\sum_{t=1}^{n}\left(F_{t}-A_{t}\right)^{2}}{n}} \\
\text { NRMSE }=\frac{\sqrt{\frac{\sum_{t=1}^{n}\left(F_{t}-A_{t}\right)^{2}}{n}} \times 100}{\bar{A}} \times 10
\end{gathered}
$$

In the experiment, Intel (R) Core (TM) i7-10700 CPU, Samsung 32 G DDR4 memory, and NVIDIA Geforce GTX 3090ti were used, and the operating system was Windows 10. Electricity load forecasting of our proposed model was performed in Python 3.7. The ensemble learning models in the first stage were constructed using scikit-learn (v.0.22.1) and tuned using a grid search [55]. The RNN-based model in the second stage was constructed using Pytorch 1.7.1 [56]. The experiments and their results for each stage are illustrated in the following subsections.

\subsection{Single-Output Forecasting Results}

In the first experiment, we compared our single-output forecasting model with other popular machine learning models. For fair comparison, TSCV was also applied to the machine learning-based forecasting models. Table 3 summarizes the hyperparameter values determined for the models by the grid search. In total, 365 days of data were used 
for initial training, while 1674 days in clusters A, B, and C, and 1430 days in cluster D, were used as test data. The comparative experimental results of single-output forecasting models are presented in Table 4.

Table 3. Selected hyperparameters for each single-output forecasting model. Selected values are bold.

\begin{tabular}{|c|c|c|c|c|}
\hline Model & Cluster A & Cluster B & Cluster C & Cluster D \\
\hline \multirow{6}{*}{ LightGBM } & Learning rate: & Learning rate: & Learning rate: & Learning rate: \\
\hline & $0.01,0.05, \mathbf{0 . 1}$ & $0.01,0.05, \mathbf{0 . 1}$ & $0.01,0.05, \mathbf{0 . 1}$ & $0.01,0.05, \mathbf{0 . 1}$ \\
\hline & No. of iterations: 500 , & No. of iterations: 500 , & No. of iterations: 500 , & No. of iterations: 500 , \\
\hline & 1000 & 1000 & 1000 & 1000 \\
\hline & No. of leaves: 64 & No. of leaves: 64 & No. of leaves: 64 & No. of leaves: 64 \\
\hline & Subsample: $\mathbf{0 . 5}, 1.0$ & Subsample: $\mathbf{0 . 5}, 1.0$ & Subsample: $\mathbf{0 . 5}, 1.0$ & Subsample: $\mathbf{0 . 5}, 1.0$ \\
\hline \multirow{7}{*}{ XGBoost } & Learning rate: $\mathbf{0 . 0 1}$ & Learning rate: $\mathbf{0 . 0 1}$ & Learning rate: 0.01 & Learning rate: 0.01 \\
\hline & $0.05,0.1$ & $0.05,0.1$ & $\mathbf{0 . 0 5}, 0.1$ & $\mathbf{0 . 0 5}, 0.1$ \\
\hline & No. of iterations: 500 , & No. of iterations: 500 , & No. of iterations: $\mathbf{5 0 0 ,}$ & No. of iterations: 500 , \\
\hline & 1000 & 1000 & 1000 & 1000 \\
\hline & Subsample: $0.5, \mathbf{1 . 0}$ & Subsample: $0.5, \mathbf{1 . 0}$ & Subsample: $\mathbf{0 . 5}, 1.0$ & Subsample: $\mathbf{0 . 5}, 1.0$ \\
\hline & Colsample by tree: & Colsample by tree: & Colsample by tree: & Colsample by tree: \\
\hline & $0.5, \mathbf{1 . 0}$ & $0.5, \mathbf{1 . 0}$ & $0.5, \mathbf{1 . 0}$ & $0.5, \mathbf{1 . 0}$ \\
\hline NGBoost & $\begin{array}{l}\text { No. of iterations: } 500 \text {, } \\
1000,1500\end{array}$ & $\begin{array}{l}\text { No. of iterations: } 500 \text {, } \\
\mathbf{1 0 0 0}, 1500\end{array}$ & $\begin{array}{l}\text { No. of iterations: } 500 \text {, } \\
1000, \mathbf{1 5 0 0}\end{array}$ & $\begin{array}{l}\text { No. of iterations: } 500 \text {, } \\
1000, \mathbf{1 5 0 0}\end{array}$ \\
\hline \multirow[b]{2}{*}{ Random Forest } & No. of trees: $64, \mathbf{1 2 8}$ & No. of trees: 64,128 & No. of trees: $64, \mathbf{1 2 8}$ & No. of trees: $64, \mathbf{1 2 8}$ \\
\hline & Random state: 32,64 & Random state: 32,64 & Random state: 32,64 & Random state: 32,64 \\
\hline \multirow{5}{*}{ MLP } & No. of layers: $4,5,6,7$ & No. of layers: $4,5,6,7$ & No. of layers: $4,5,6,7$ & No. of layers: $4,5,6,7$ \\
\hline & Activation function: & Activation function: & Activation function: & Activation function: \\
\hline & ReLU & ReLU & ReLU & ReLU \\
\hline & Optimizer: Adam & Optimizer: Adam & Optimizer: Adam & Optimizer: Adam \\
\hline & Learning rate: $\mathbf{0 . 0 0 1}$ & Learning rate: $\mathbf{0 . 0 0 1}$ & Learning rate: $\mathbf{0 . 0 0 1}$ & Learning rate: $\mathbf{0 . 0 0 1}$ \\
\hline
\end{tabular}

Table 4. The comparative experimental results of single-output forecasting models.

\begin{tabular}{|c|c|c|c|c|c|}
\hline Evaluation Metric & Model & Cluster A & Cluster B & Cluster C & Cluster D \\
\hline \multirow{5}{*}{ MAPE (\%) } & LightGBM & 7.01 & 4.74 & 6.98 & 4.98 \\
\hline & MLP & 12.06 & 7.03 & 7.61 & 5.67 \\
\hline & RF & 7.53 & 4.98 & 7.24 & 5.38 \\
\hline & XGBoost & 7.22 & 5.12 & 7.52 & 5.29 \\
\hline & NGBoost & 9.64 & 5.44 & 7.73 & 5.90 \\
\hline \multirow{5}{*}{ MAE (kWh) } & LightGBM & 40.71 & 32.00 & 22.18 & 23.47 \\
\hline & MLP & 68.59 & 45.95 & 23.10 & 27.30 \\
\hline & RF & 43.81 & 34.09 & 23.46 & 25.49 \\
\hline & XGBoost & 41.81 & 32.81 & 24.76 & 24.81 \\
\hline & NGBoost & 54.25 & 37.04 & 24.85 & 29.21 \\
\hline \multirow{5}{*}{ RMSE (kWh) } & LightGBM & 61.31 & 49.01 & 30.81 & 36.26 \\
\hline & MLP & 119.07 & 75.39 & 32.5 & 41.11 \\
\hline & RF & 67.2 & 54.73 & 32.89 & 40.51 \\
\hline & XGBoost & 63.58 & 48.34 & 34.39 & 37.16 \\
\hline & NGBoost & 82.05 & 59.1 & 34.43 & 44.17 \\
\hline \multirow{5}{*}{ NRMSE (\%) } & LightGBM & 9.37 & 7.63 & 9.78 & 7.25 \\
\hline & MLP & 18.91 & 11.74 & 10.32 & 8.22 \\
\hline & RF & 10.67 & 8.52 & 10.45 & 8.10 \\
\hline & XGBoost & 10.09 & 7.53 & 10.92 & 7.43 \\
\hline & NGBoost & 13.03 & 9.21 & 10.93 & 8.83 \\
\hline
\end{tabular}

The experimental results show that the LightGBM model outperformed other forecasting models in all metrics in most clusters. Therefore, we used the LightGBM forecasting 
values as the new input variable for the second stage. Table 5 presents the PCC between the single-output forecasting results of LightGBM and the actual electricity loads, which indicates a strong correlation between them. Therefore, we use a single-output prediction value for the second stage input to improve the MSA forecasting performance.

Table 5. PCC between single-output forecasting and actual electricity loads.

\begin{tabular}{cccc}
\hline Cluster A & Cluster B & Cluster C & Cluster D \\
\hline 0.984 & 0.978 & 0.923 & 0.957 \\
\hline
\end{tabular}

\subsection{Multistep-Ahead Forecasting Results}

In order to evaluate the performance of the proposed model, we carried out extensive experiments with various MSA forecasting models. As mentioned earlier, in the experiments, we used data from January 2016 to December 2018 of clusters A, B, and C and data from September 2016 to December 2018 of cluster D as the training set, and data from January 2019 to July 2020 as the test set. To evaluate the validity of our forecasting model, we compared it with basic deep learning, ensemble learning-based MSA forecasting models, and other models including ATT-GRU [30], DALSTM [33], and COSMOS [34]. The ensemble learning-based models require all input variables for 96 forecasting time points for MSA forecasting. Therefore, we used 1824 input variables (i.e., 19 input variables $\times 96$ forecasting points). The ensemble learning-based MSA forecasting models perform predictions on multiple outputs using the MultiOutputRegressor module in scikit-learn. The hyperparameter configurations of comparative forecasting models are presented in Table 6 .

Table 6. Selected hyperparameters for each multistep-ahead forecasting model.

\begin{tabular}{ccc}
\hline Model & Package & Selected Hyperparameters \\
\hline \multirow{2}{*}{ LightGBM } & Learning rate: 0.05 \\
& No. of iterations: 1000 \\
& No. of leaves: 32 \\
Random Forest & Subsample: 0.5 \\
\hline \multirow{2}{*}{ Scikit-learn } & No. of trees: 128 \\
& Random state: 64 \\
\hline \multirow{2}{*}{ S2S BiLSTM } & Pytorch & No. of hidden nodes: 15 \\
& & No. of hidden layers: 2 \\
& & Activation function: ReLU \\
& Optimizer: Adam \\
& Learning rate: 0.001 \\
& No. of epochs: 350 \\
\hline \multirow{2}{*}{ S2S ATT-BiLSTM } & Po. of hidden nodes: 15 \\
& & No. of hidden layers: 2 \\
& & Activation function: ReLU \\
& Optimizer: Adam \\
& & Learning rate: 0.001 \\
& & No. of epochs: 350 \\
\hline & & No. of hidden nodes: 15 \\
& & No. of hidden layers: 2 \\
& & Octivation function: SELU \\
& Optimizer: Adam \\
& & Learning rate: 0.001 \\
& & No. of epochs: 150 \\
\hline & &
\end{tabular}


Table 6. Cont.

\begin{tabular}{|c|c|c|}
\hline Model & Package & Selected Hyperparameters \\
\hline \multirow{2}{*}{$\begin{array}{c}\text { DALSTM [33] } \\
\text { Stage 1: LSTM } \\
\text { Stage 2: DARNN }\end{array}$} & \multirow{2}{*}{ Pytorch } & $\begin{array}{c}\text { LSTM } \\
\text { No. of hidden nodes: } 15 \\
\text { No. of hidden layers: } 2 \\
\text { Activation function: ReLU } \\
\text { Optimizer: Adam } \\
\text { Learning rate: } 0.001 \\
\text { No. of epochs: } 350\end{array}$ \\
\hline & & $\begin{array}{c}\text { DARNN } \\
\text { No. of hidden nodes: } 64 \\
\text { Time steps: } 96 \\
\text { Optimizer: Adam } \\
\text { Learning rate: } 0.001 \\
\text { No. of epochs: } 150\end{array}$ \\
\hline \multirow[t]{2}{*}{$\begin{array}{l}\text { COSMOS [34] } \\
\text { Stage 1: MLP } \\
\text { Stage 2: PCR }\end{array}$} & \multirow[t]{2}{*}{ Scikit-learn } & $\begin{array}{c}\text { MLP } \\
\text { No. of hidden nodes: } 15 \\
\text { No. of hidden layers: } 4,5,6,7 \\
\text { Activation function: ReLU } \\
\text { Optimizer: Adam } \\
\text { Learning rate: } 0.001 \\
\text { No. of epochs: } 150\end{array}$ \\
\hline & & $\begin{array}{c}\text { PCR } \\
\text { Principal components: } 1 \\
\text { Sliding window size: } 672\end{array}$ \\
\hline
\end{tabular}

Figures 9 and 10 illustrate the average of the four evaluation metrics of the forecasting models for each cluster over the entire forecasting horizon. For instance, we can see that the proposed model achieved the best NRMSE and MAPE across all clusters, with one exception, as shown in Figure 9. That is, the LightGBM-based forecasting model achieved the best NRMSE in cluster A with a narrow margin compared to the proposed model. Similarly, the proposed model achieved the best performance for MAE and RMSE across all clusters, with one exception, as shown in Figure 10. The LightGBM-based forecasting model achieved the best RMSE in cluster A with a narrow margin compared to the proposed model. Furthermore, from the evaluation results of S2S BiLSTM, S2S ATTBiLSTM, and the proposed model, it can be seen that attention and two-stage forecasting can improve the forecasting performance. Figures 11-18 show the trends of the metric values of the forecasting models over the entire forecasting horizon for clusters A, B, C and $D$. In the figures, the $X$-axis represents forecasting time points, and the $Y$-axis represents their forecasting errors.

Figures 11 and 12 illustrate the MAPE results for the four clusters over the entire forecasting horizon. They show that the proposed model outperforms the other models for all clusters. Similarly, Figures 13 and 14 show that the proposed model achieves the best NRMSE for all clusters except cluster A. On the other hand, Figures 15 and 16 present the MAE results for the four clusters, and the proposed model shows the best performance out of all clusters. Lastly, Figures 17 and 18 illustrate the RMSE results, and the proposed model shows the best performance for all clusters except cluster A. 


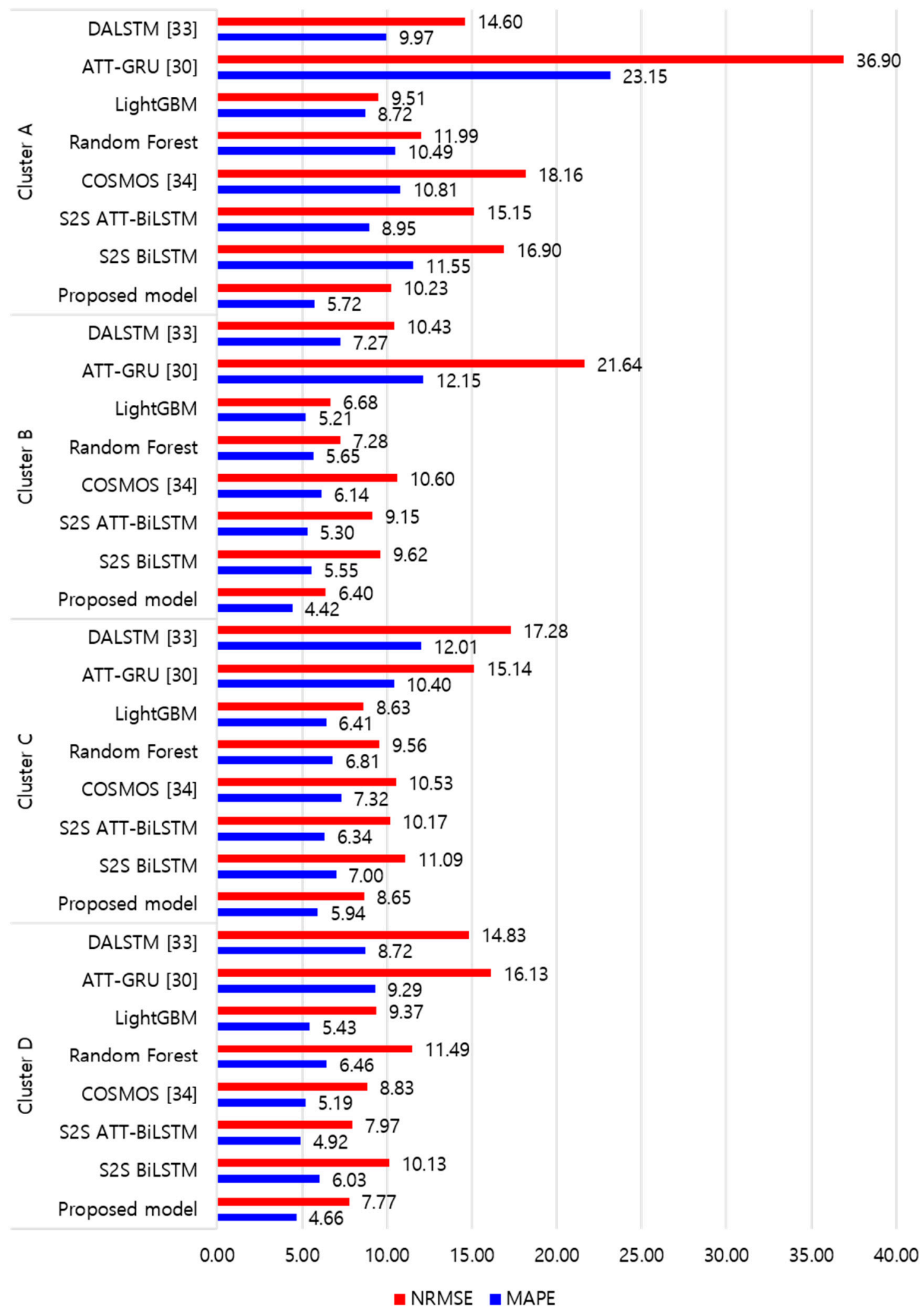

Figure 9. Average of NRMSE and MAPE for each model (\%). 


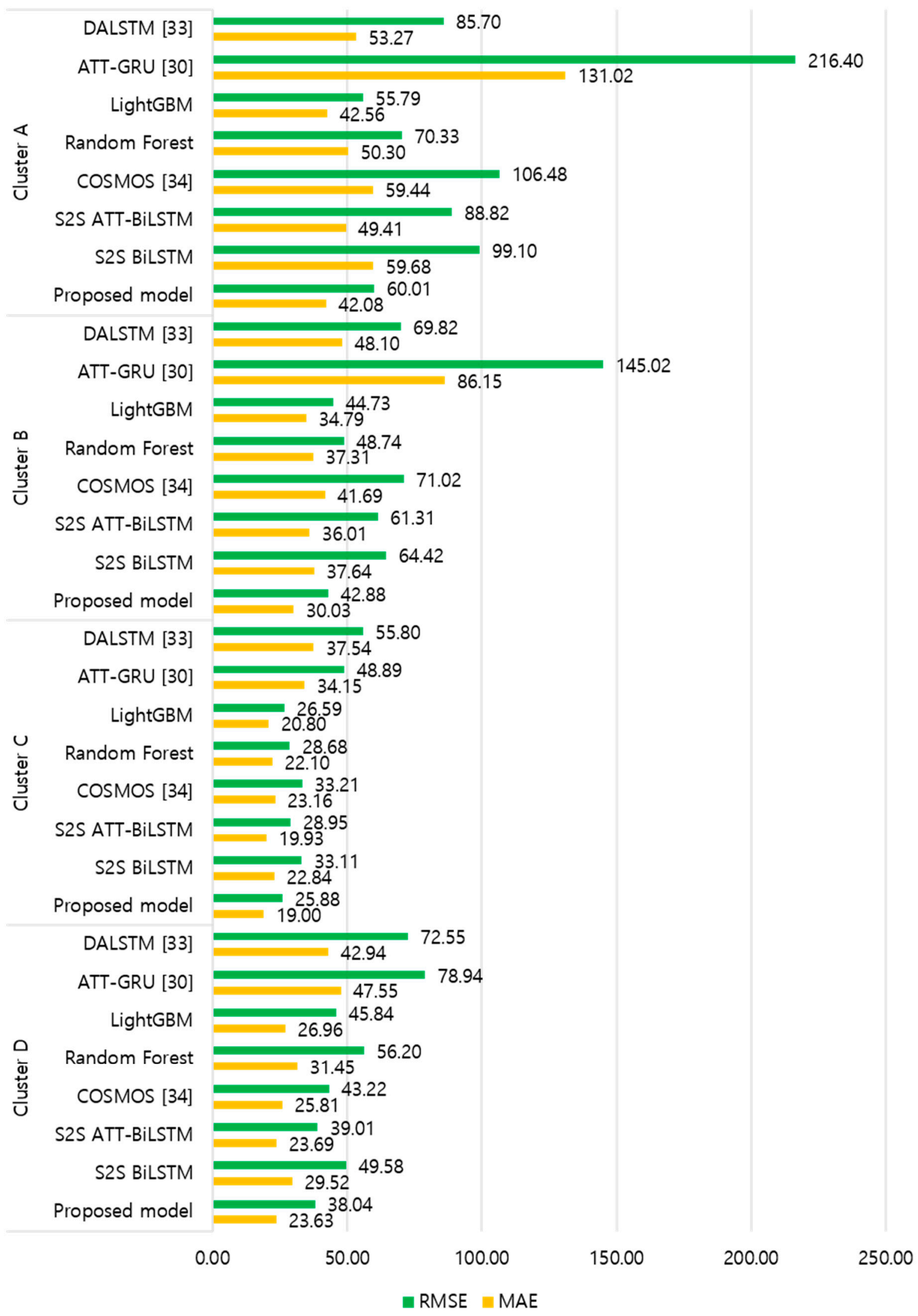

Figure 10. Average of RMSE and MAE for each model (kWh). 

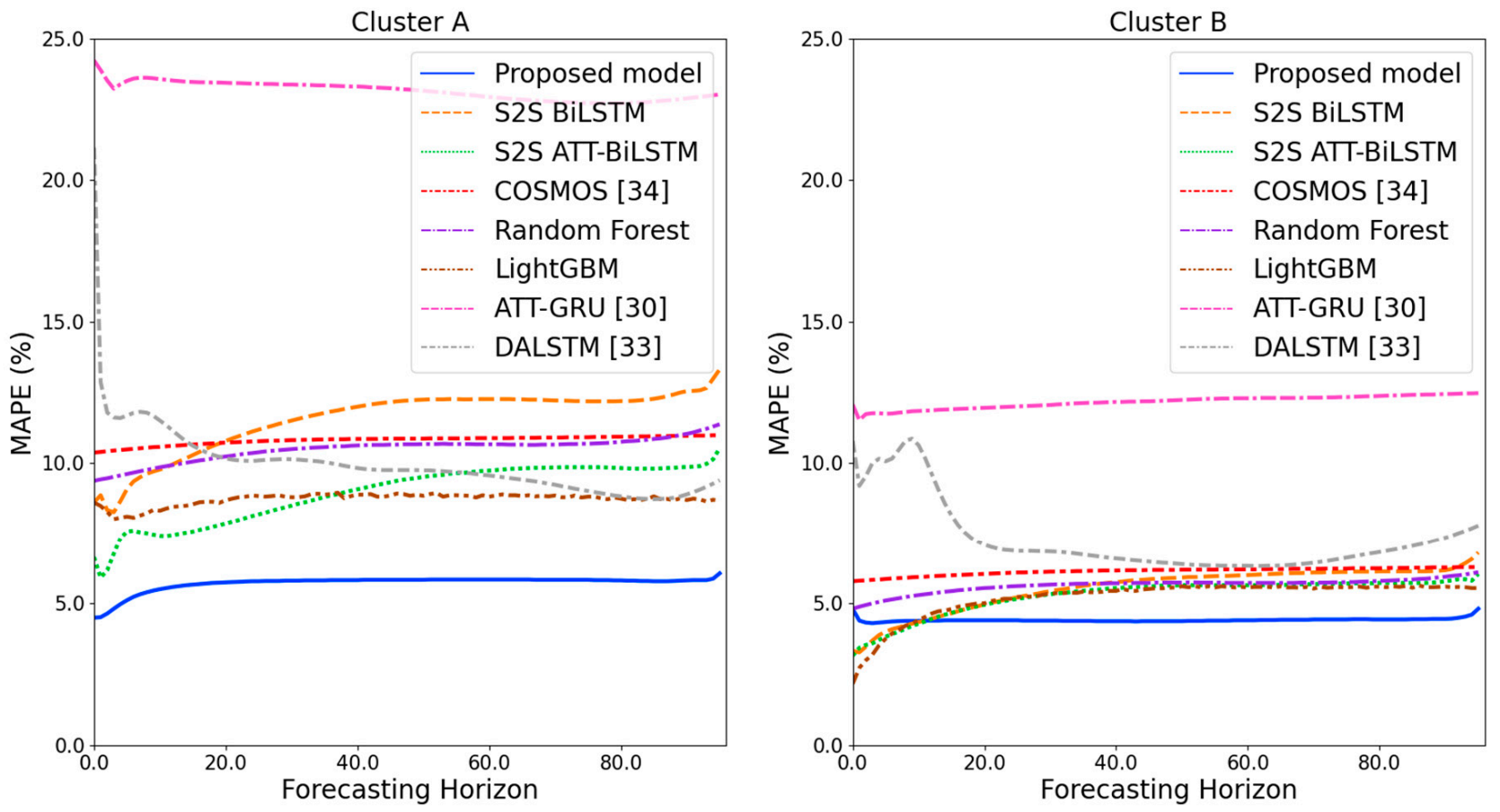

Figure 11. MAPE results of clusters A and B over the entire forecasting horizon in (\%).
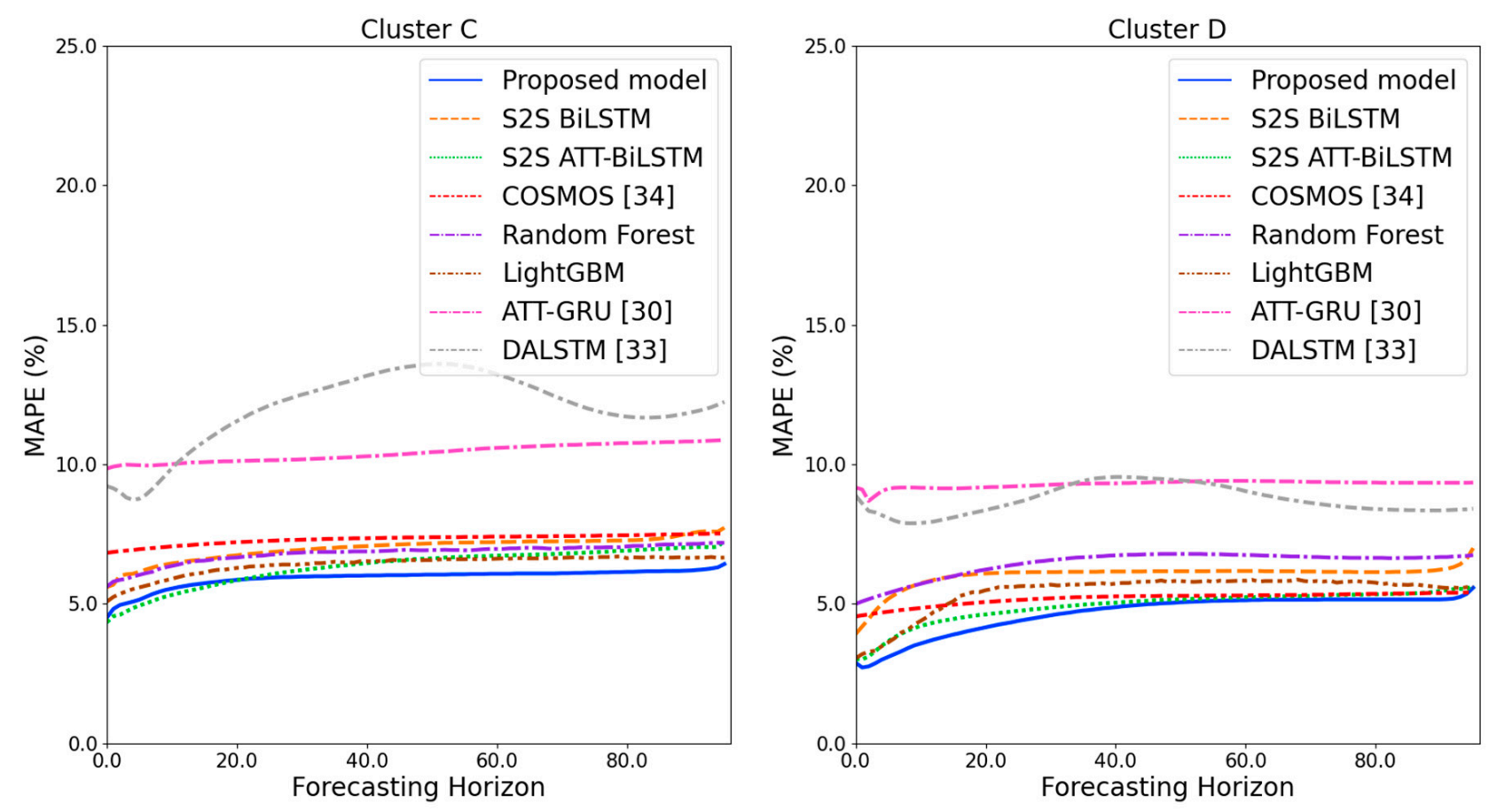

Figure 12. MAPE results of clusters $C$ and D over the entire forecasting horizon (\%). 

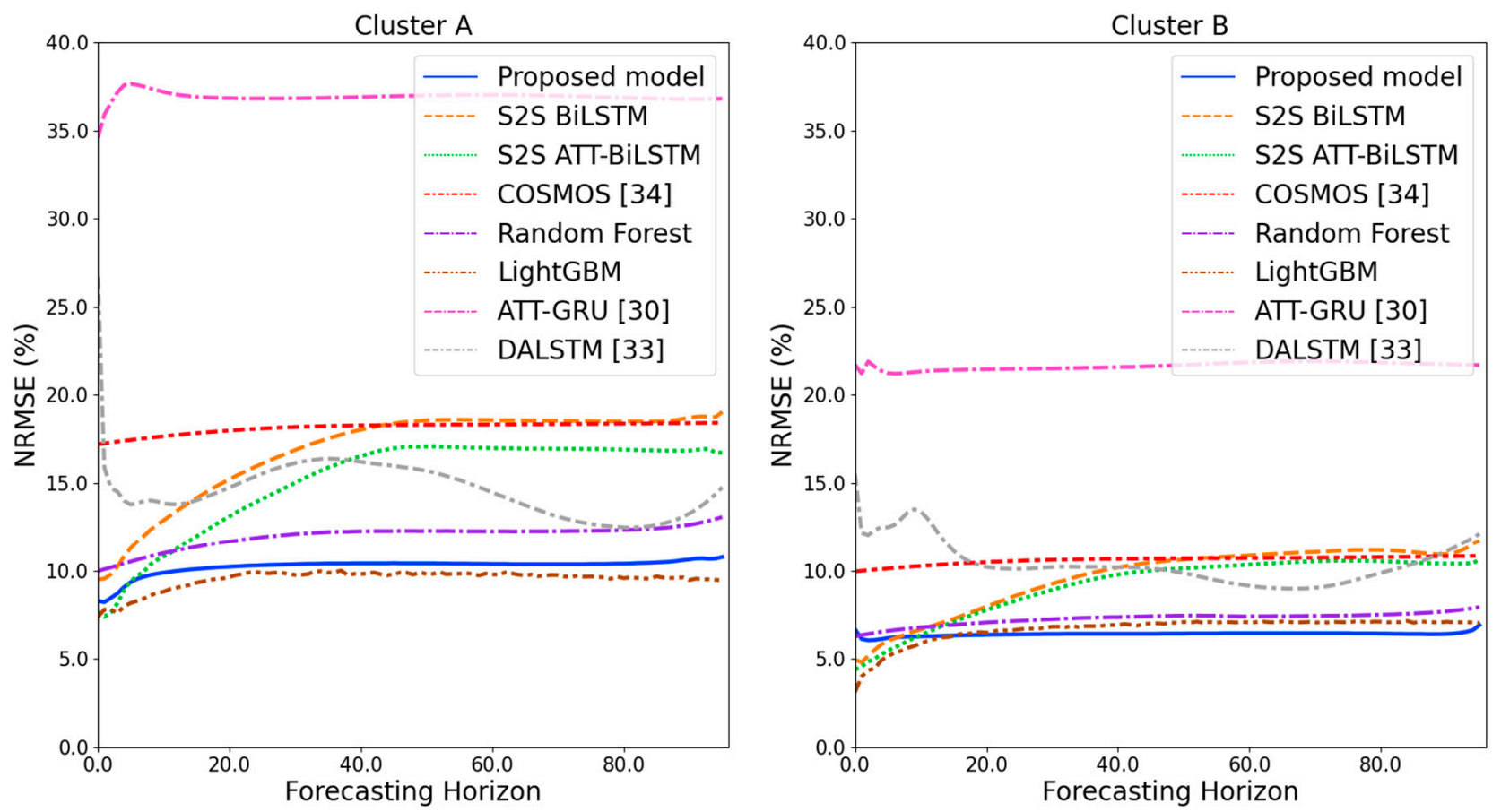

Figure 13. NRMSE results of clusters A and B over the entire forecasting horizon (\%).
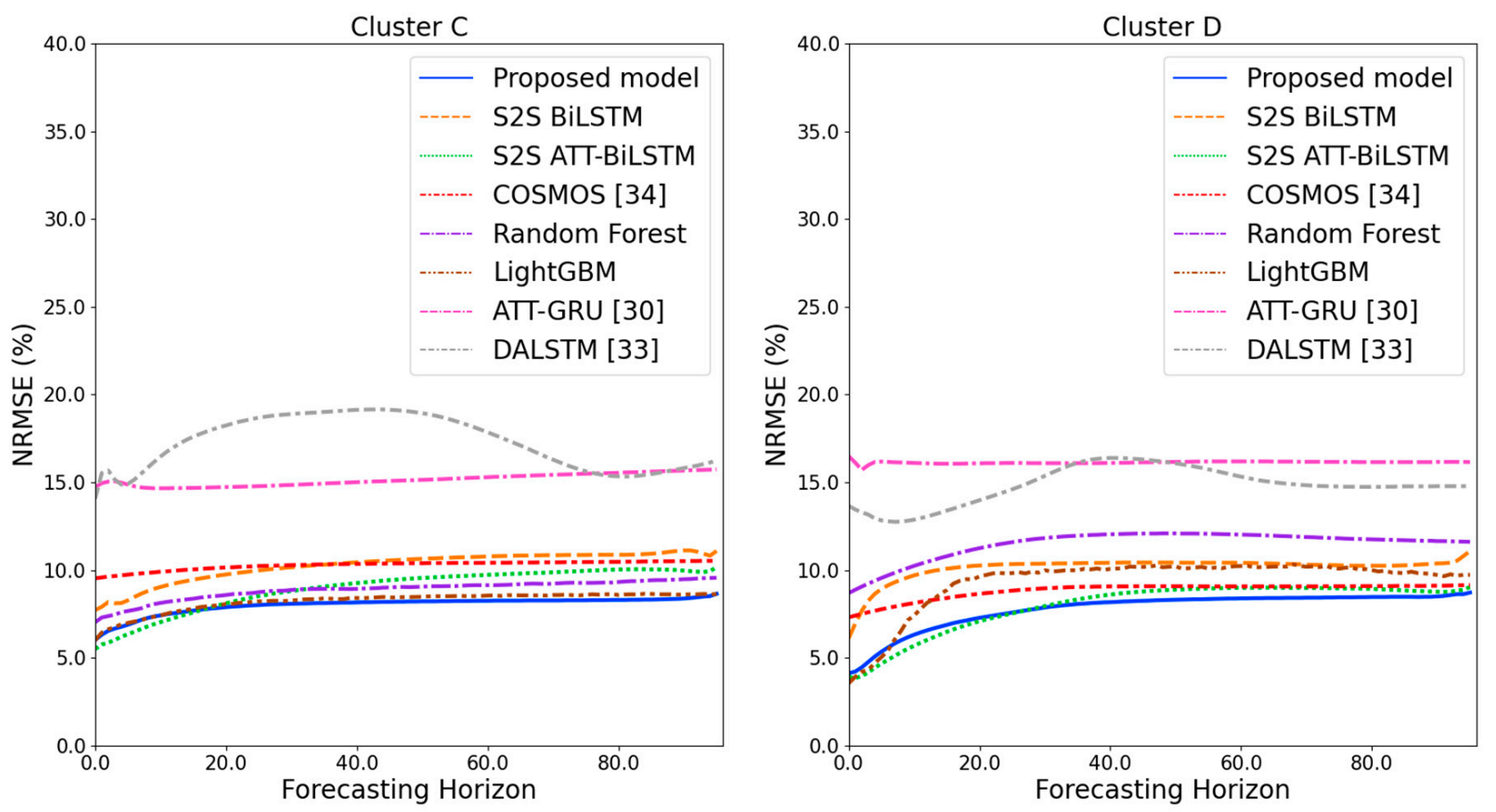

Figure 14. NRMSE results of clusters C and D over the entire forecasting horizon (\%). 

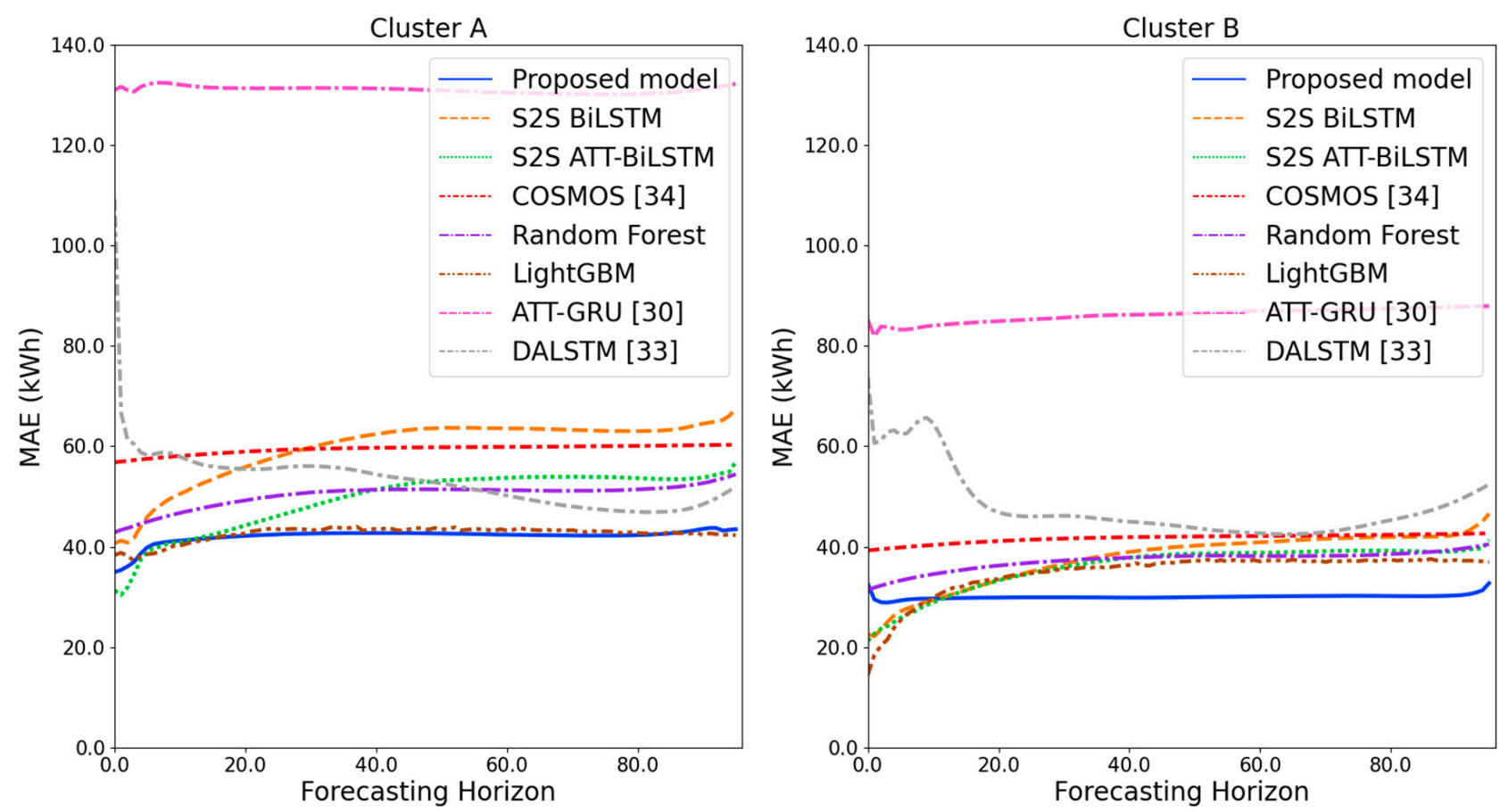

Figure 15. MAE results of clusters $A$ and B over the entire forecasting horizon $(\mathrm{kWh})$.
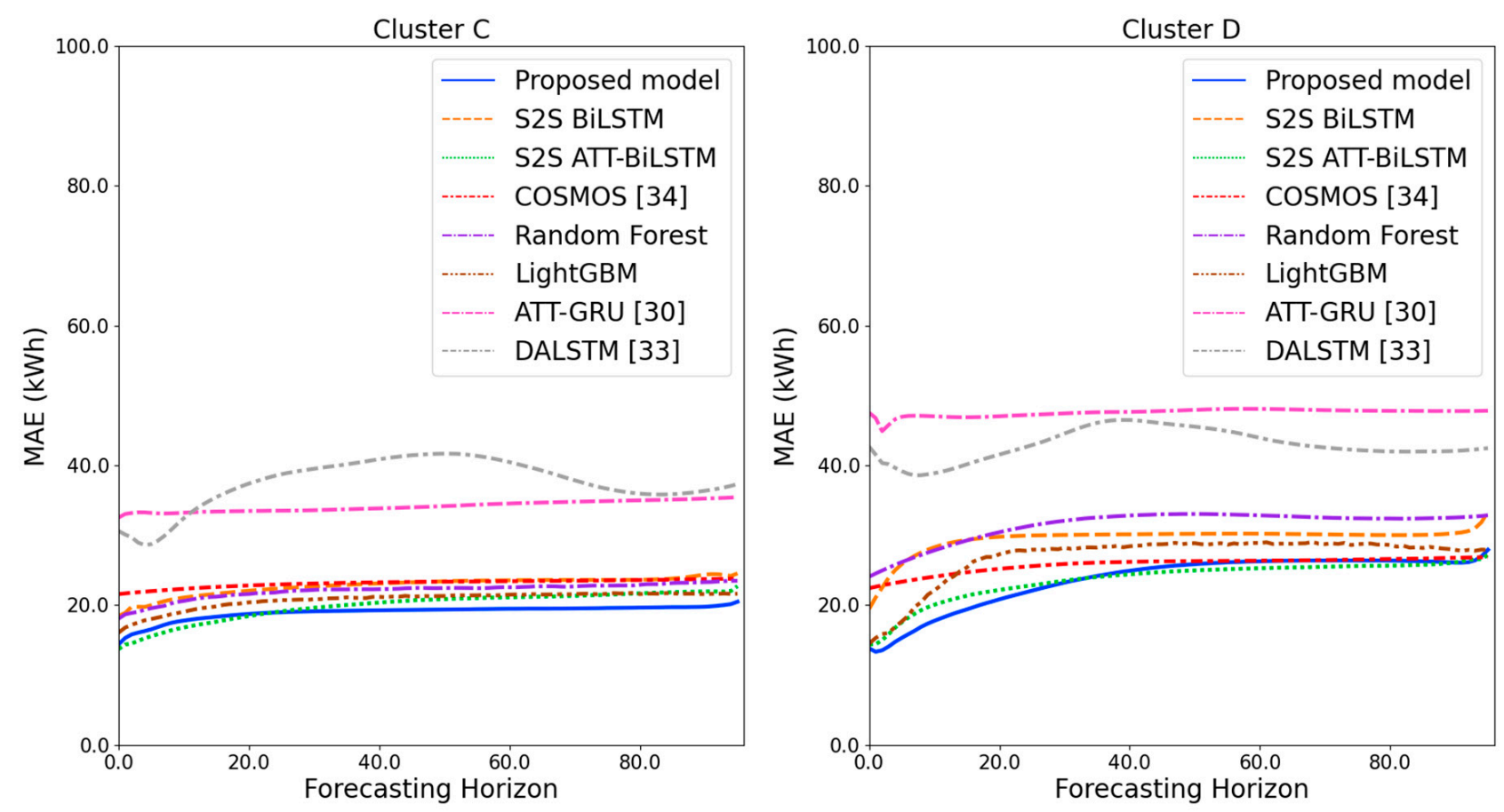

Figure 16. MAE results of clusters $C$ and D over the entire forecasting horizon $(\mathrm{kWh})$. 

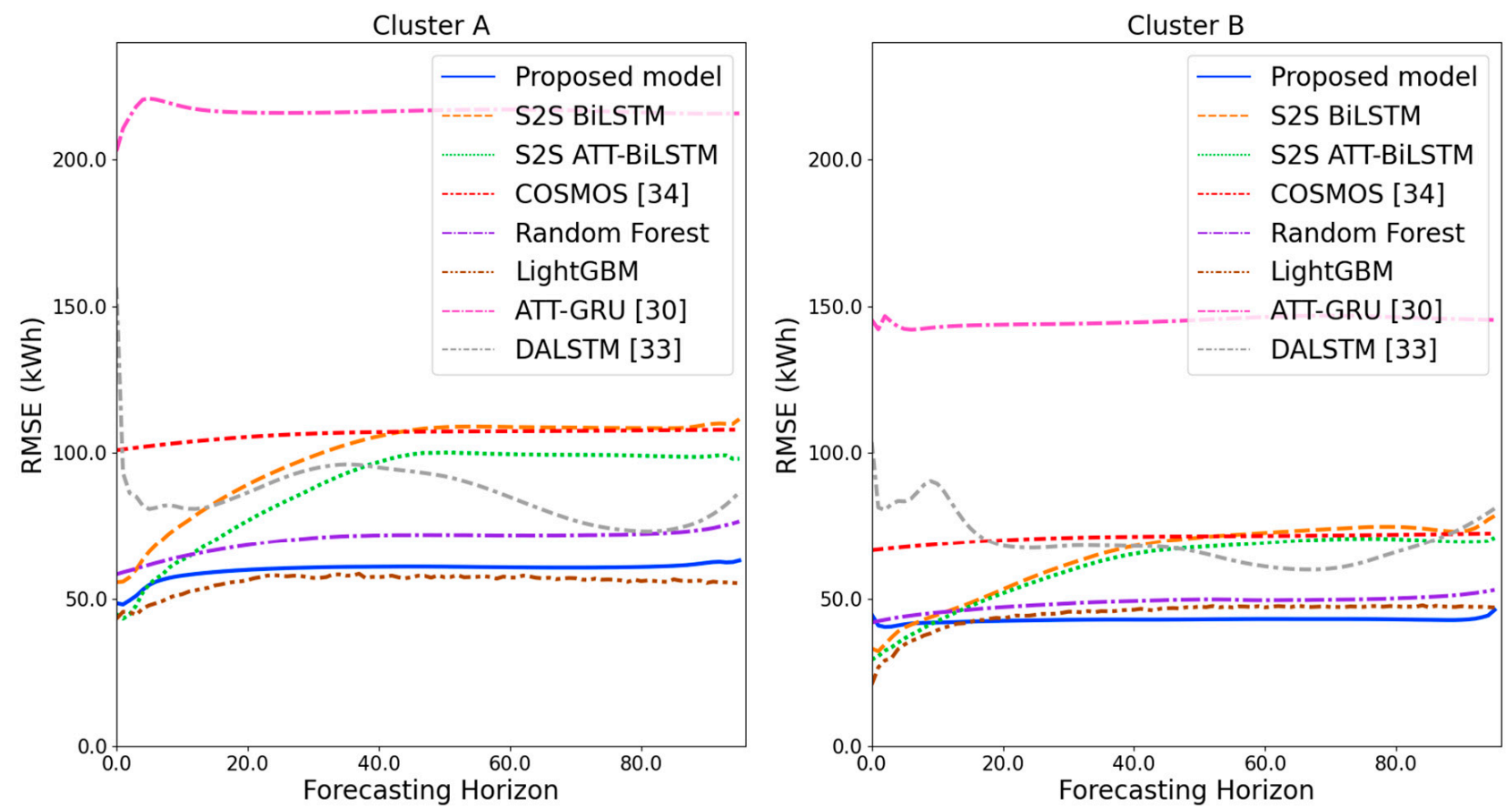

Figure 17. RMSE results of clusters A and B over the entire forecasting horizon (kWh).
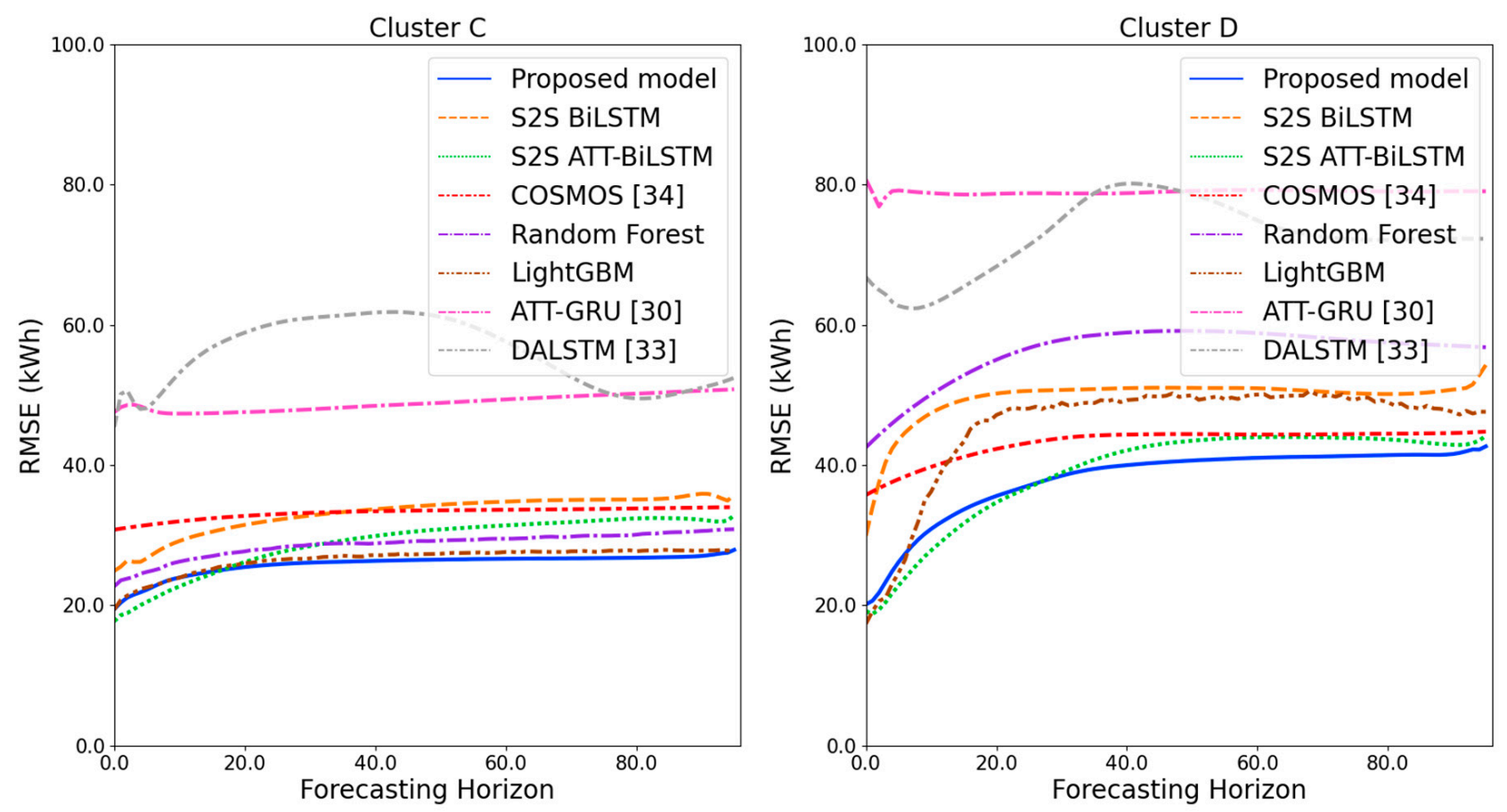

Figure 18. RMSE results of clusters $C$ and D over the entire forecasting horizon (kWh).

From the figures, we can see that the proposed model gives very stable and highquality prediction performance in terms of four evaluation metrics over the entire forecasting horizon for all clusters, compared to other forecasting models. On the other hand, ATT-GRU [30] shows poor prediction performance because it cannot reflect a lot of information due to using a single network. Similarly, DALSTM [33] has poor forecasting accuracy in the second stage because the DARNN model is unsuitable for multistep-ahead forecasting. On the other hand, COSMOS [34] showed stable performance, as it used only the predicted values as inputs for MSA forecasting. However, its performance was 
greatly affected by the first-stage prediction. The only exception was the LightGBM-based forecasting model, which gave slightly better average RMSE and NRMSE for cluster A only. Furthermore, we compared the proposed model with the S2SATT-BiLSTM-based model to show that the proposed model combined with a single-output prediction can reliably predict over the entire forecasting horizon. In addition, we compared the S2S ATTBiLSTM- and S2S BiLSTM-based models to verify the influence of attention mechanisms on prediction accuracy.

\section{Conclusions}

In this paper, we proposed a robust two-stage MSA forecasting model that combines a single-output forecasting model and a MSA forecasting model. To show the effectiveness of the proposed model, we conducted extensive comparative experiments with other popular forecasting models using electricity load data of four types of building clusters at a private university. Overall, the experimental results showed that the forecasting performance can be improved by combining the two models and by using an attention mechanism. Nevertheless, the proposed model cannot explain the evidence of the output properly. Hence, in future work, we plan to implement explainable artificial intelligence through attention score analysis of attention mechanism. We will also investigate how to optimize hyperparameters to improve forecasting performance.

Author Contributions: Conceptualization, J.P.; methodology, J.P.; software, J.P.; validation, J.P.; formal analysis, J.P.; investigation, J.P.; data curation, J.P.; writing-original draft preparation, J.P.; writing-review and editing, E.H.; visualization, J.P.; supervision, E.H.; project administration, E.H.; funding acquisition, E.H. All authors have read and agreed to the published version of the manuscript.

Funding: This research was supported by Energy Cloud R\&D Program (grant number: 2019M3F2A10 73184) through the National Research Foundation of Korea (NRF) funded by the Ministry of Science and ICT.

Institutional Review Board Statement: Not applicable.

Informed Consent Statement: Not applicable.

Data Availability Statement: Not applicable.

Conflicts of Interest: The authors declare no conflict of interest.

\begin{tabular}{|c|c|}
\hline Abbrev & \\
\hline The follov & bbreviations are used in this manuscript \\
\hline ICT & information and communications technology \\
\hline STLF & Short-term load forecasting \\
\hline AI & artificial intelligence \\
\hline MSA & multistep-ahead \\
\hline RNN & recurrent neural network \\
\hline LSTM & long short-term memory \\
\hline GRU & gated recurrent unit \\
\hline S2S & sequence-to-sequence \\
\hline ANN & artificial neural network \\
\hline ARIMA & autoregressive integrated moving average \\
\hline MLR & multiple linear regression \\
\hline PCR & principal component regression \\
\hline PCC & Pearson correlation coefficient \\
\hline WCI & windchill index \\
\hline GBM & gradient boosting machine \\
\hline ReLU & rectified linear unit \\
\hline MAE & mean absolute error \\
\hline RMSE & root mean square error \\
\hline NN & neural network \\
\hline DARNN & dual-stage attention-based recurrent neural network \\
\hline
\end{tabular}




$\begin{array}{ll}\text { ATT-GRU } & \text { attention-based gated recurrent unit } \\ \text { LightGBM } & \text { light gradient boosting machine } \\ \text { TSCV } & \text { Time series cross-validation } \\ \text { BiLSTM } & \text { bidirectional long short-term memory } \\ \text { ATT-BiLSTM } & \text { bidirectional long short-term memory with attention mechanism } \\ \text { SVR } & \text { support vector regression } \\ \text { RF } & \text { random forest } \\ \text { FIR } & \text { fuzzy inductive reasoning } \\ \text { MLP } & \text { multilayer perceptron } \\ \text { CNN } & \text { convolutional neural network } \\ \text { XGB } & \text { extreme gradient boosting } \\ \text { ML } & \text { machine learning } \\ \text { KMA } & \text { Korea meteorological administration } \\ \text { DI } & \text { discomfort index } \\ \text { GBDT } & \text { gradient boosting decision tree } \\ \text { BA } & \text { Bahdanau attention mechanism } \\ \text { Adam } & \text { adaptive moment estimation } \\ \text { MAPE } & \text { mean absolute percentage error } \\ \text { NRMSE } & \text { normalized root mean square error } \\ \text { RICNN } & \text { recurrent inception convolution neural network } \\ \text { DALSTM } & \text { dual-stage attentional long short-term memory } \\ \text { COSMOS } & \text { combination of short-term load forecasting models using a stacking ensemble approach }\end{array}$

\section{References}

1. Atef, S.; Eltawil, A.B. Assessment of stacked unidirectional and bidirectional long short-term memory networks for electricity load forecasting. Electr. Power Syst. Res. 2020, 187, 106489. [CrossRef]

2. Hong, T.; Fan, S. Probabilistic electric load forecasting: A tutorial review. Int. J. Forecast. 2016, 32, 914-938. [CrossRef]

3. Li, B.W.; Zhang, J.; He, Y.; Wang, Y. Short-Term Load-Forecasting Method Based on Wavelet Decomposition with Second-Order Gray Neural Network Model Combined with ADF Test. IEEE Access 2017, 5, 16324-16331. [CrossRef]

4. Rana, M.; Koprinska, I. Forecasting electricity load with advanced wavelet neural networks. Neurocomputing 2016, 182, 118-132. [CrossRef]

5. Dong, Y.X.; Ma, X.J.; Fu, T.L. Electrical load forecasting: A deep learning approach based on K-nearest neighbors. Appl. Soft Comput. 2021, 99, 106900. [CrossRef]

6. Dodamani, S.; Shetty, V.; Magadum, R. Short term load forecast based on time series analysis: A case study. In Proceedings of the 2015 International Conference on Technological Advancements in Power and Energy (TAP Energy), Kollam, India, 24-26 June 2015; pp. 299-303.

7. Song, K.-B.; Baek, Y.-S.; Hong, D.H.; Jang, G. Short-term load forecasting for the holidays using fuzzy linear regression method. IEEE Trans. Power Syst. 2005, 20, 96-101. [CrossRef]

8. Taylor, J.W.; McSharry, P.E. Short-term load forecasting methods: An evaluation based on European data. IEEE Trans. Power Syst. 2007, 22, 2213-2219. [CrossRef]

9. Kelo, S.; Dudul, S. A wavelet Elman neural network for short-term electrical load prediction under the influence of temperature. Int. J. Electr. Power Energy Syst. 2012, 43, 1063-1071. [CrossRef]

10. Zhang, Z.C.; Hong, W.C.; Li, J.C. Electric Load Forecasting by Hybrid Self-Recurrent Support Vector Regression Model with Variational Mode Decomposition and Improved Cuckoo Search Algorithm. IEEE Access 2020, 8, 14642-14658. [CrossRef]

11. Chen, Y.B.; Xu, P.; Chu, Y.Y.; Li, W.L.; Wu, Y.T.; Ni, L.Z.; Bao, Y.; Wang, K. Short-term electrical load forecasting using the Support Vector Regression (SVR) model to calculate the demand response baseline for office buildings. Appl. Energy 2017, 195, 659-670. [CrossRef]

12. Yu, F.; Xu, X.Z. A short-term load forecasting model of natural gas based on optimized genetic algorithm and improved BP neural network. Appl. Energy 2014, 134, 102-113. [CrossRef]

13. Yeom, C.U.; Kwak, K.C. Short-Term Electricity-Load Forecasting Using a TSK-Based Extreme Learning Machine with Knowledge Representation. Energies 2017, 10, 1613. [CrossRef]

14. Liu, T.X.; Zhao, Q.J.; Wang, J.Z.; Gao, Y.Y. A novel interval forecasting system for uncertainty modeling based on multi-input multi-output theory: A case study on modern wind stations. Renew. Energy 2021, 163, 88-104. [CrossRef]

15. Pei, S.Q.; Qin, H.; Yao, L.Q.; Liu, Y.Q.; Wang, C.; Zhou, J.Z. Multi-Step Ahead Short-Term Load Forecasting Using Hybrid Feature Selection and Improved Long Short-Term Memory Network. Energies 2020, 13, 4121. [CrossRef]

16. Sehovac, L.; Nesen, C.; Grolinger, K. Forecasting building energy consumption with deep learning: A sequence to sequence approach. In Proceedings of the 2019 IEEE International Congress on Internet of Things (ICIOT), Milan, Italy, 8-13 July 2019; pp. 108-116. 
17. Jarábek, T.; Laurinec, P.; Lucká, M. Energy load forecast using S2S deep neural networks with k-Shape clustering. In Proceedings of the 2017 IEEE 14th International Scientific Conference on Informatics, Poprad, Slovakia, 14-16 November 2017; pp. 140-145.

18. Bahdanau, D.; Cho, K.; Bengio, Y. Neural machine translation by jointly learning to align and translate. arXiv 2014, arXiv:1409.0473.

19. Luong, M.-T.; Pham, H.; Manning, C.D. Effective approaches to attention-based neural machine translation. arXiv 2015, arXiv:1508.04025.

20. Sehovac, L.; Grolinger, K. Deep Learning for Load Forecasting: Sequence to Sequence Recurrent Neural Networks with Attention. IEEE Access 2020, 8, 36411-36426. [CrossRef]

21. Gollou, A.R.; Ghadimi, N. A new feature selection and hybrid forecast engine for day-ahead price forecasting of electricity markets. J. Intell. Fuzzy Syst. 2017, 32, 4031-4045. [CrossRef]

22. Jalili, A.; Ghadimi, N. Hybrid Harmony Search Algorithm and Fuzzy Mechanism for Solving Congestion Management Problem in an Electricity Market. Complexity 2016, 21, 90-98. [CrossRef]

23. Fan, G.F.; Peng, L.L.; Hong, W.C.; Sun, F. Electric load forecasting by the SVR model with differential empirical mode decomposition and auto regression. Neurocomputing 2016, 173, 958-970. [CrossRef]

24. Grolinger, K.; L'Heureux, A.; Capretz, M.A.M.; Seewald, L. Energy Forecasting for Event Venues: Big Data and Prediction Accuracy. Energy Build. 2016, 112, 222-233. [CrossRef]

25. Jurado, S.; Nebot, A.; Mugica, F.; Avellana, N. Hybrid methodologies for electricity load forecasting: Entropy-based feature selection with machine learning and soft computing techniques. Energy 2015, 86, 276-291. [CrossRef]

26. Zhang, X.B.; Wang, J.Z.; Zhang, K.Q. Short-term electric load forecasting based on singular spectrum analysis and support vector machine optimized by Cuckoo search algorithm. Electr. Power Syst. Res. 2017, 146, 270-285. [CrossRef]

27. Zheng, J.; Xu, C.; Zhang, Z.; Li, X. Electric load forecasting in smart grids using long-short-term-memory based recurrent neural network. In Proceedings of the 2017 51st Annual Conference on Information Sciences and Systems (CISS), Baltimore, MD, USA, 22-24 March 2017; pp. 1-6.

28. Marino, D.L.; Amarasinghe, K.; Manic, M. Building energy load forecasting using deep neural networks. In Proceedings of the IECON 2016-42nd Annual Conference of the IEEE Industrial Electronics Society, Florence, Italy, 23-26 October 2016; pp. 7046-7051.

29. Kim, J.; Moon, J.; Hwang, E.; Kang, P. Recurrent inception convolution neural network for multi short-term load forecasting. Energy Build. 2019, 194, 328-341. [CrossRef]

30. Jung, S.; Moon, J.; Park, S.; Hwang, E. An Attention-Based Multilayer GRU Model for Multistep-Ahead Short-Term Load Forecasting (dagger). Sensors 2021, 21, 1639. [CrossRef] [PubMed]

31. Kuo, P.-H.; Huang, C.-J. A high precision artificial neural networks model for short-term energy load forecasting. Energies 2018, 11, 213. [CrossRef]

32. Park, S.; Moon, J.; Jung, S.; Rho, S.; Baik, S.W.; Hwang, E. A Two-Stage Industrial Load Forecasting Scheme for Day-Ahead Combined Cooling, Heating and Power Scheduling. Energies 2020, 13, 443. [CrossRef]

33. Siridhipakul, C.; Vateekul, P. Multi-step power consumption forecasting in Thailand using dual-stage attentional LSTM. In Proceedings of the 2019 11th International Conference on Information Technology and Electrical Engineering (ICITEE), Pattaya, Thailand, 10-11 October 2019; pp. 1-6.

34. Moon, J.; Jung, S.; Rew, J.; Rho, S.; Hwang, E. Combination of short-term load forecasting models based on a stacking ensemble approach. Energy Build. 2020, 216, 109921. [CrossRef]

35. Nie, H.; Liu, G.; Liu, X.; Wang, Y. Hybrid of ARIMA and SVMs for short-term load forecasting. Energy Procedia 2012, 16, 1455-1460. [CrossRef]

36. Tian, C.; Ma, J.; Zhang, C.; Zhan, P. A deep neural network model for short-term load forecast based on long short-term memory network and convolutional neural network. Energies 2018, 11, 3493. [CrossRef]

37. Xie, Y.; Ueda, Y.; Sugiyama, M. A Two-Stage Short-Term Load Forecasting Method Using Long Short-Term Memory and Multilayer Perceptron. Energies 2021, 14, 5873. [CrossRef]

38. Oliveira, M.O.; Marzec, D.P.; Bordin, G.; Bretas, A.S.; Bernardon, D. Climate change effect on very short-term electric load forecasting. In Proceedings of the 2011 IEEE Trondheim PowerTech, Trondheim, Norway, 19-23 June 2011; pp. 1-7.

39. Park, J.; Moon, J.; Jung, S.; Hwang, E. Multistep-Ahead Solar Radiation Forecasting Scheme Based on the Light Gradient Boosting Machine: A Case Study of Jeju Island. Remote Sens. 2020, 12, 2271. [CrossRef]

40. Ke, G.; Meng, Q.; Finley, T.; Wang, T.; Chen, W.; Ma, W.; Ye, Q.; Liu, T.-Y. LightGBM: A highly efficient gradient boosting decision tree. Adv. Neural Inf. Process. Syst. 2017, 30, 3146-3154.

41. Wang, Y.; Chen, J.; Chen, X.; Zeng, X.; Kong, Y.; Sun, S.; Guo, Y.; Liu, Y. Short-term load forecasting for industrial customers based on TCN-LightGBM. IEEE Trans. Power Syst. 2020, 36, 1984-1997. [CrossRef]

42. Park, S.; Jung, S.; Jung, S.; Rho, S.; Hwang, E. Sliding window-based LightGBM model for electric load forecasting using anomaly repair. J. Supercomput. 2021, 77, 12857-12878. [CrossRef]

43. Huang, H.; Jia, R.; Liang, J.; Dang, J.; Wang, Z. Wind Power Deterministic Prediction and Uncertainty Quantification Based on Interval Estimation. J. Sol. Energy Eng. 2021, 143, 061010. [CrossRef]

44. De Livera, A.M.; Hyndman, R.J.; Snyder, R.D. Forecasting time series with complex seasonal patterns using exponential smoothing. J. Am. Stat. Assoc. 2011, 106, 1513-1527. [CrossRef]

45. Moon, J.; Kim, Y.; Son, M.; Hwang, E. Hybrid Short-Term Load Forecasting Scheme Using Random Forest and Multilayer Perceptron. Energies 2018, 11, 3283. [CrossRef] 
46. Werbos, P.J. Backpropagation through Time-What It Does and How to Do It. Proc. IEEE 1990, 78, 1550-1560. [CrossRef]

47. Pascanu, R.; Mikolov, T.; Bengio, Y. On the difficulty of training recurrent neural networks. Proc. Int. Conf. Mach. Learn. 2013, 28, 1310-1318.

48. Hochreiter, S.; Schmidhuber, J. Long short-term memory. Neural Comput. 1997, 9, 1735-1780. [CrossRef] [PubMed]

49. Robinson, A.J. An Application of Recurrent Nets to Phone Probability Estimation. IEEE T Neural Netw. 1994, 5, 298-305. [CrossRef] [PubMed]

50. Schuster, M.; Paliwal, K.K. Bidirectional recurrent neural networks. IEEE Trans. Signal Process. 1997, 45, 2673-2681. [CrossRef]

51. Sutskever, I.; Vinyals, O.; Le, Q.V. Sequence to sequence learning with neural networks. arXiv 2014, arXiv:1409.3215.

52. Nair, V.; Hinton, G.E. Rectified linear units improve restricted Boltzmann machines. In Proceedings of the Icml, Haifa, Israel, 21-24 June 2010.

53. Huber, P.J. Robust estimation of a location parameter. In Breakthroughs in Statistics; Springer: Berlin/Heidelberg, Germany, 1992; pp. 492-518.

54. Kingma, D.P.; Ba, J. Adam: A method for stochastic optimization. arXiv 2014, arXiv:1412.6980.

55. Pedregosa, F.; Varoquaux, G.; Gramfort, A.; Michel, V.; Thirion, B.; Grisel, O.; Blondel, M.; Prettenhofer, P.; Weiss, R.; Dubourg, V. Scikit-learn: Machine learning in Python. J. Mach. Learn. Res. 2011, 12, 2825-2830.

56. Paszke, A.; Gross, S.; Massa, F.; Lerer, A.; Bradbury, J.; Chanan, G.; Killeen, T.; Lin, Z.; Gimelshein, N.; Antiga, L. Pytorch: An imperative style, high-performance deep learning library. arXiv 2019, arXiv:1912.01703. 